\title{
Mutation-Specific Phenotypes in hiPSC-Derived Cardiomyocytes Carrying Either Myosin-Binding Protein C Or $\alpha$-Tropomyosin Mutation for Hypertrophic Cardiomyopathy
}

\author{
Marisa Ojala, ${ }^{1}$ Chandra Prajapati, ${ }^{1}$ Risto-Pekka Pölönen, ${ }^{1}$ Kristiina Rajala, ${ }^{1}$ \\ Mari Pekkanen-Mattila, ${ }^{1}$ Jyrki Rasku, ${ }^{2}$ Kim Larsson, ${ }^{1}$ and Katriina Aalto-Setälä ${ }^{1,3,4}$ \\ ${ }^{1}$ BioMediTech, University of Tampere, 33014 Tampere, Finland \\ ${ }^{2}$ School of Information Sciences, University of Tampere, 33014 Tampere, Finland \\ ${ }^{3}$ Medical School, University of Tampere, 33014 Tampere, Finland \\ ${ }^{4}$ Heart Hospital, Tampere University Hospital, 33521 Tampere, Finland \\ Correspondence should be addressed to Katriina Aalto-Setälä; katriina.aalto-setala@uta.fi
}

Received 21 May 2015; Revised 22 July 2015; Accepted 20 September 2015

Academic Editor: Farah Sheikh

Copyright ( 2016 Marisa Ojala et al. This is an open access article distributed under the Creative Commons Attribution License, which permits unrestricted use, distribution, and reproduction in any medium, provided the original work is properly cited.

\begin{abstract}
Hypertrophic cardiomyopathy (HCM) is a genetic cardiac disease, which affects the structure of heart muscle tissue. The clinical symptoms include arrhythmias, progressive heart failure, and even sudden cardiac death but the mutation carrier can also be totally asymptomatic. To date, over 1400 mutations have been linked to HCM, mostly in genes encoding for sarcomeric proteins. However, the pathophysiological mechanisms of the disease are still largely unknown. Two founder mutations for HCM in Finland are located in myosin-binding protein C (MYBPC3-Gln1061X) and $\alpha$-tropomyosin (TPM1-Asp175Asn) genes. We studied the properties of HCM cardiomyocytes (CMs) derived from patient-specific human induced pluripotent stem cells (hiPSCs) carrying either MYBPC3-Gln1061X or TPM1-Asp175Asn mutation. Both types of HCM-CMs displayed pathological phenotype of HCM but, more importantly, we found differences between CMs carrying either MYBPC3-Gln1061X or TPM1-Asp175Asn gene mutation in their cellular size, $\mathrm{Ca}^{2+}$ handling, and electrophysiological properties, as well as their gene expression profiles. These findings suggest that even though the clinical phenotypes of the patients carrying either MYBPC3-Gln1061X or TPM1-Asp175Asn gene mutation are similar, the genetic background as well as the functional properties on the cellular level might be different, indicating that the pathophysiological mechanisms behind the two mutations would be divergent as well.
\end{abstract}

\section{Introduction}

Hypertrophic cardiomyopathy (HCM) is one of the most common genetic cardiac diseases with worldwide prevalence of 1:500, as well as the most common cause of sudden cardiac death (SCD) among young competing athletes. HCM is inherited in an autosomal dominant pattern. Nevertheless, a large clinical diversity and age-related penetrance are typical for HCM. On the tissue level, HCM is characterized by the disarray of cardiomyocytes (CMs) and fibrosis of cardiac tissue, as well as thickened interventricular septum or free left ventricular wall. Clinical symptoms include arrhythmias, progressive heart failure, and even SCD, but on the other hand the mutation carrier can be completely asymptomatic.
Altogether more than 1400 mutations in 11 genes encoding for the sarcomeric proteins have been identified and related to HCM. The majority of the mutations are found either in the $\beta$-myosin heavy chain (MYH7) or in the myosin-binding protein C (MYBPC3) genes [1]. In Finland, two founder mutations located in MYBPC3 and $\alpha$-tropomyosin (TPM1) genes and one common mutation in $M Y H 7$ gene together account around $24 \%$ of all Finnish HCM cases [2, 3].

Although the genetic information related to HCM has been growing in the recent years due to the development of sequencing technologies, exact information of the disease mechanisms remains unclear. Thus, current medication of the disease is directed toward the symptom relief and there is no specific therapy to prevent the onset or progression of the 
disease [1]. Most of the HCM studies have been conducted with model systems, mainly either with transgenic mice or by studying human tissues obtained from surgical myectomy from end-stage HCM patients [4]. However, animal models carry only the mutated gene lacking the rest of the genome and myectomy samples are obtained from patients in the late stage of HCM development. Therefore, the discovery of the human induced pluripotent stem cells (hiPSCs) has offered a new valuable tool to model HCM and other cardiac diseases and to study the underlying disease mechanisms [5]. To date, hiPSCs have already been used to model a variety of cardiac diseases: electrical defects, for example, long-QT syndrome [6-8] and catecholaminergic polymorphic ventricular tachycardia (CPVT) $[9,10]$ as well as cardiomyopathies including dilated cardiomyopathy (DCM) [11] and HCM [12-14].

Here we have derived hiPSCs from patients carrying two of the Finnish HCM founder mutations either in MYBPC3 (MYBPC3-Gln1061X) or in TPM1 (TPM1-Asp175Asn) gene. We have differentiated the patient-specific hiPSCs into CMs and compared the phenotypes of the diseased and control CMs.

\section{Materials and Methods}

2.1. Ethical Issues. This study was conducted in accordance with the Ethics Committee of Pirkanmaa Hospital District to establish, culture, and differentiate hiPSC lines (R08070). Skin biopsies for hiPSC establishment were received from the Heart Hospital, Tampere University Hospital, Tampere, Finland. Patients donating skin biopsies signed an informed consent after receiving both oral and written descriptions of the study. The teratoma assay, described in Section 2.3.6, was approved by ELLA-Animal Experiment Board of Regional State Administrative Agency for Southern Finland (ESAVI/6543/04.10.03/2011).

2.2. Generation and Culture of Patient-Specific hiPSC Lines. hiPSC lines were generated from skin's fibroblasts either with Sendai reprogramming vectors OCT4, KLF4, c-MYC, and SOX2 using CytoTune-iPS Reprogramming Kit (Life Technologies Ltd., Paisley, UK) according to the manufacturer's instructions or by using pMX retroviral vectors OCT4, KLF4, $c-M Y C$, and SOX2 with or without Cre-LoxP site as described earlier [6, 15]. UTA.13602.HCMT, UTA.02912.HCMT, and UTA.04511.WT hiPSC lines were generated by using Sendai vectors and UTA.07801.HCMM and UTA.06108.HCMM by using pMX retroviral vectors with Cre-LoxP site and UTA.04602.WT was generated by using pMX retroviral vectors without Cre-LoxP site. In the present study, one line of each patient was used. hiPSC lines were derived and cultured on mouse embryonic fibroblast (MEF) feeder cell layers (26000 cells $/ \mathrm{cm}^{2}$, CellSystems Biotechnologie Vertrieb $\mathrm{GmbH}$, Troisdorf, Germany) in human pluripotent stem cell (hPSC) culture medium consisting of knockout-DMEM (koDMEM, Gibco, Life Technologies Ltd.) supplemented with $20 \%$ knockout serum replacement (ko-SR, Gibco, Life Technologies Ltd.), 1\% nonessential amino acids (NEAA, Lonza Group Ltd., Basel, Switzerland), 2 mM GlutaMax (Gibco, Life
Technologies Ltd.), $50 \mathrm{U} / \mathrm{mL}$ penicillin/streptomycin (Lonza Group Ltd.), 0.1 mM 2-mercaptoethanol (Gibco, Life Technologies Ltd.), and $4 \mathrm{ng} / \mathrm{mL}$ basic fibroblast growth factor (bFGF, PeproTech, Rocky Hill, NJ, USA).

\subsection{Characterization of hiPSC Lines}

2.3.1. Mutation Analysis by Genotyping. DNA samples from the hiPSC lines were prepared with TaqMan Sample-toSNP Kit (Applied Biosystems, Life Technologies Ltd.) and the presence of MYBPC3-Gln1061X and TPM1-Asp175Asn mutation in the patient-specific hiPSC lines was confirmed by custom TaqMan SNP Genotyping Assays (Applied Biosystems, Life Technologies Ltd.) according to the manufacturer's instructions. In the genotyping assays, MYBPC3-gene as well as TPM1-gene was amplified with specific primers. Furthermore, the presence of the mutations was assessed with mutation-specific FAM labeled probes. VIC labeled probes were used to assess the presence of the wild type allele. Sequences for the primers and probes used in the assay are listed in Supplementary Table 1 (see Supplementary Material available online at http://dx.doi.org/10.1155/2016/1684792).

2.3.2. The Expression of Mutant and Wild Type Alleles in hiPSC-Derived CMs. RNA samples were collected and extracted from hiPSC-derived CMs (UTA.04511.WT, UTA.02912.HCMT, UTA.07801.HCMM, and UTA.06108. HCMM) with Norgen's Total RNA Purification Plus Kit (Norgen Biotek Corp., Ontario, Canada) according to manufacturer's instructions. 50-100 ng of RNA was transcribed to cDNA by High-Capacity cDNA Reverse Transcription Kit (Applied Biosystems, Life Technologies Ltd.). The expression of TPM1-Asp175Asn or MYBPC3-Gln1061X mutation on mRNA level in the hiPSC-derived CMs was studied by Custom TaqMan SNP Genotyping Assays (Applied Biosystems, Life Technologies Ltd.) similarly as that for genotyping described above. Sequences for the primers and probes used in the assay are listed in Supplementary Table 1.

2.3.3. Immunocytochemistry. Undifferentiated hiPSC colonies were fixed with $4 \%$ paraformaldehyde (PFA, SigmaAldrich, Saint Louis, USA), stained with primary antibodies for Nanog (R\&D systems Inc., Minneapolis, MN, USA), OCT4 (R\&D systems Inc.), SOX2 (Santa Cruz Biotechnology, Santa Cruz, CA, USA), TRA-1-60 (Millipore, Billerica, MA, USA), and TRA-1-81 (Millipore), and visualized with secondary antibodies as described before [16]. Finally, the cells were mounted with Vectashield (Vector Laboratories Inc., Burlingame, CA, USA) containing 40,6-diamidino-2phenylindole (DAPI) for the nuclei staining and imaged with an Olympus IX51 phase contrast microscope equipped with fluorescence optics and Olympus DP30BW camera (Olympus Corporation, Hamburg, Germany).

2.3.4. $R T-P C R$. The RNA was extracted from the hiPSC lines by NucleoSpin RNA II Kit (Macherey-Nagel GmbH \& Co., Düren, Germany) and 500-1000 ng of RNA was transcribed to cDNA by High-Capacity cDNA Reverse Transcription Kit 
(Applied Biosystems, Life Technologies Ltd.). The presence of pluripotency genes Nanog, SOX2, REX1, OCT4, and c-MYC and the absence of virally imported exogenes (OCT4, SOX2, $c-M Y C$, and KLF4) were confirmed by RT-PCR. GAPDH was used as an endogenous control. The primer sequences for pluripotency genes and virally imported exogenes have been published earlier [5]. The primer sequences used for detection of Sendai transgenes are described in CytoTune-iPS Reprogramming Kit's manual (Life Technologies Ltd.).

2.3.5. Karyotype Analysis. The karyotypes of hiPSC lines were studied by G-banding (Medix Laboratories, Espoo, Finland) or by KaryoLite assay [17] (Turku Centre for Biotechnology, University of Turku, Turku, Finland).

2.3.6. Pluripotency Analysis. The pluripotency of hiPSC lines was confirmed in vitro by embryoid body (EB) formation and in vivo by teratoma assay. hiPSCs were removed from feeder cell layer and cultured in suspension to form EBs. The EBs were cultured in EB medium consisting of ko-DMEM supplemented with $20 \%$ fetal bovine serum (FBS, Biosera, Boussens, France), 1\% NEAA (Lonza Group Ltd.), $2 \mathrm{mM}$ GlutaMax (Invitrogen, Life Technologies Ltd.), and $50 \mathrm{U} / \mathrm{mL}$ penicillin/streptomycin (Lonza Group Ltd) for 4-6 weeks before RNA extraction. $200 \mathrm{ng}$ of RNA was transcribed to cDNA for the RT-PCR analysis. The presence of all three germ layers, endoderm (AFP, SOX17), ectoderm (SOX1, NESTIN, and $M$ usashi), and mesoderm (KDR, alpha cardiac actin), was studied with RT-PCR.

For in vivo pluripotency assay, hiPSCs were injected under the testis capsule of nude mice and the formed teratomas were collected and fixed with $4 \%$ PFA 8 weeks after the injection. Teratomas were embedded in paraffin, cut in sections, and stained with haematoxylin and eosin.

2.4. Differentiation of Cardiomyocytes. hiPSCs were differentiated into CMs by coculturing with mouse visceral endodermal-like cells (END-2) (Hubrecht Institute, Utrecht, Netherlands) as described before [18]. After 15-30 days beating areas were cut from cocultures and dissociated into single cells in EB medium by Collagenase A (Roche Diagnostics, Mannheim, Germany) as described earlier [18] and plated to $0.1 \%$ gelatin-coated cover slips or well plates for further analysis.

\subsection{Characterization of hiPSC-Derived Cardiomyocytes}

2.5.1. Immunocytochemistry and Image Analysis. Dissociated CMs were fixed with 4\% PFA and stained with Troponin T (cTnT, 1:2000, ab64623, Abcam, Cambridge, MA, USA), MYBPC (1:400, sc-166081, Santa Cruz Biotechnology), and TPM1 (1:200, sc-73225, Santa Cruz Biotechnology) primary antibodies, followed by labeling with secondary antibodies. Images were obtained with Olympus IX51 phase contrast microscope equipped with fluorescence optics and Olympus DP308W camera (Olympus Corporation) or with Zeiss AxioScope A1 fluorescent microscope and Zeiss AxioCam MRc5 camera (Carl Zeiss, Jena, Germany). Size of the Troponin T stained CMs was analyzed from 46 to $50 \mathrm{CMs}$ in each cell line by in-house made software (unpublished method). CMs were analyzed from pictures obtained with Olympus IX51 phase contrast microscope. The proportion of multinucleated CMs was determined from the same images (46-50 CMs/cell line).

2.5.2. $\mathrm{Ca}^{2+}$ Imaging. The clusters of CMs were cut, dissociated, plated on $0.1 \%$ gelatin-coated coverslips, and cultured for 1,3 , and 6 weeks. To study the $\mathrm{Ca}^{2+}$ handling properties of hiPSC-derived CMs, cells were loaded with $4 \mu \mathrm{M}$ Fura- $2 \mathrm{AM}$ (Molecular Probes, Life Technologies Ltd.) for 30 minutes in perfusate medium. The perfusate medium consisted of (in $\mathrm{mM}$ ) $137 \mathrm{NaCl}, 5 \mathrm{KCl}, 0.44 \mathrm{KH}_{2} \mathrm{PO}_{4}, 20 \mathrm{HEPES}, 4.2$ $\mathrm{NaHCO}_{3}, 5 \mathrm{D}$-glucose, $2 \mathrm{CaCl}_{2}, 1.2 \mathrm{MgCl}_{2}$, and $1 \mathrm{Na}$-pyruvate dissolved in $\mathrm{H}_{2} \mathrm{O}$. $\mathrm{pH}$ of the perfusate medium was adjusted to 7.4 with $\mathrm{NaOH}$. The coverslip, containing the dissociated hiPSC-derived CMs, was mounted to an RC-25 recording chamber and continuously perfused with perfusate medium preheated to $35-36^{\circ} \mathrm{C}$ by an $\mathrm{SH}-27 \mathrm{~B}$ inline-heater controlled by a TC-324B unit (all from Warner Instruments Inc., Hamden, USA). The perfusion was controlled by a gravity driven $\mathrm{VC}^{3} 8$ application system (ALA Scientific Instruments Inc., NY, USA). Coverslip was perfused for 15 minutes for Fura-2 AM deesterification before experimental recordings. $\mathrm{Ca}^{2+}$ handling of spontaneously beating CMs was imaged with an inverted IX70 microscope using UApo/340 x20 air objective (Olympus Corporation) and ANDOR iXon 885 CCD camera (Andor Technology, Belfast, Northern Ireland) synchronized with a Polychrome V light source by a real time DPS control unit. TILLvisION or Live Acquisition software (TILL Photonics, Munich, Germany) was used for recording. Fura-2 AM was excited at $340 \mathrm{~nm}$ and $380 \mathrm{~nm}$ light and the emission was recorded for 10-30 seconds at $505 \mathrm{~nm}$.

For $\mathrm{Ca}^{2+}$ imaging analysis, single beating CMs were selected as regions of interests and background noise, recorded from a cell-free area in the same coverslip, was subtracted before further processing. Data is presented as ratios of 340/380 $\mathrm{nm}$ (F340/F380). The spontaneously beating CMs were divided into five different rhythm categories based on the abnormalities observed in their $\mathrm{Ca}^{2+}$ signals: normal beating with regular peaks (normal); more than three peaks which do not return to the baseline (oscillation); signals with small or middle sized amplitude events in the beginning, in the end, or in between two $\mathrm{Ca}^{2+}$ spikes (low/middle peaks); two or three peaks which do not return to the baseline (double peaks); $\mathrm{Ca}^{2+}$ spikes with prolonged rise or decay time (plateau abnormality). In the low/middle peaks category, the small amplitude was at least $10 \%$ from the preceding $\mathrm{Ca}^{2+}$ spike amplitude. Full-length, 10-30 seconds long recordings were analyzed, while most of the analyzed recordings were 12 seconds long. The distribution of CMs in different categories is presented for each cell lines separately.

2.5.3. Electrophysiological Measurements: Recording and Analysis of Action Potentials. The action potentials (APs) were recorded by conventional patch clamp [19] in perforated patch configuration using Amphotericin B [20] in final concentration of $0.24 \mathrm{mg} / \mathrm{mL}$ [8]. Data acquisition was 
TABLE 1: TaqMan assays used in qRT-PCR experiments.

\begin{tabular}{|c|c|c|c|}
\hline Gene & Description/alias & Function & TaqMan assay ID \\
\hline EEF1A1 & Eukaryotic translation elongation factor 1 alpha 1 & Housekeeping gene & Hs00265885_g1 \\
\hline GAPDH & Glyceraldehyde-3-phosphate dehydrogenase & Housekeeping gene & Hs02758991_g1 \\
\hline TNNT2 & Troponin $\mathrm{T}$ & Sarcomeric gene & Hs00165960_ml \\
\hline MYH6 & Myosin heavy chain 6 & Sarcomeric gene & Hs01101425_m1 \\
\hline ACTN2 & $\alpha$-actinin 2 & Sarcomeric gene & Hs00153809_m1 \\
\hline TPM1 & $\alpha$-tropomyosin & Sarcomeric gene & Hs00165966_ml \\
\hline MYBPC3 & Myosin-binding protein $\mathrm{C}$ & Sarcomeric gene & Hs00165232_ml \\
\hline TTN & Titin & Sarcomeric gene & Hs00399225_ml \\
\hline TNNC1 & Troponin C type 1 & Sarcomeric gene & Hs00896999_g1 \\
\hline MYL9 & Myosin, light chain 2/MLC2 & Sarcomeric gene & Hs00697086_ml \\
\hline MYL7 & Myosin, light chain 7 & Sarcomeric gene & Hs01085598_g1 \\
\hline$N P P A$ & Natriuretic peptide A & Hypertrophy marker & Hs01081097_m1 \\
\hline$N P P B$ & Natriuretic peptide B & Hypertrophy marker & Hs01057466_g1 \\
\hline $\mathrm{HCN} 4$ & Hyperpolarization activated cyclic nucleotide-gated potassium channel 4 & Potassium channel & Hs00975492_m1 \\
\hline KCNQ1 & Voltage-gated potassium channel, KQT-like subfamily, member 1 & Potassium channel & Hs00923522_m1 \\
\hline CACNA1C & Voltage-dependent calcium channel, L type, alpha 1C subunit/CaCNA1.2 & Calcium channel & Hs00167681_m1 \\
\hline SCN5A & Voltage-gated sodium channel, $\mathrm{V}$ type, alpha subunit & Sodium channel & Hs00165693_ml \\
\hline SLC8A1 & Solute carrier family 8 , member $1 / \mathrm{NCX} 1$ & $\mathrm{Na}^{+} / \mathrm{Ca}^{2+}$ exchanger & Hs01062258_ml \\
\hline$P L N$ & Phospholamban/PLB & Protein kinase substrate & Hs01848144_s1 \\
\hline ATP2A2 & ATPase, $\mathrm{Ca}^{2+}$ transporting, cardiac muscle, slow twitch 2/SERCA2a & $\mathrm{Ca}^{2+}$-ATPase & Hs00544877_ml \\
\hline CASQ2 & Calsequestrin & $\mathrm{Ca}^{2+}$ binding protein in $\mathrm{SR}$ & Hs00154286_ml \\
\hline ITPR2 & Inositol 1,4,5-trisphosphate receptor, type 2/IP3R2 & $\mathrm{Ca}^{2+}$ receptor & Hs00181916_m1 \\
\hline RYR2 & Ryanodine receptor 2 (cardiac) & Ryanodine receptor & Hs00892883_ml \\
\hline
\end{tabular}

conducted using Axon Series 200B patch-clamp amplifier connected to Digidata 1440a AD/DA converter driven by pCLAMP 10.2 software (all from Molecular devices LLC). On the day of use, the coverslips containing dissociated hiPSC-derived CMs were transferred to RC-24N recording chamber (Warner Instruments Inc.) and mounted on an inverted Olympus IX71 microscope (Olympus Corporation). The patch electrodes had tip resistance of 3.0-3.5 M $\Omega$ and contained the following intracellular solution (in $\mathrm{mM}$ ): 132 $\mathrm{KMeSO}_{4}, 20 \mathrm{KCl}, 1 \mathrm{MgCl}_{2}$, and $1 \mathrm{CaCl}_{2}$ (pH was adjusted to 7.2 with $\mathrm{KOH}$ ). The extracellular solution contained (in $\mathrm{mM}$ ) $143 \mathrm{NaCl}, 4.8 \mathrm{KCl}, 1.8 \mathrm{CaCl}_{2}, 1.2 \mathrm{MgCl}_{2}, 5$ glucose, and 10 HEPES (pH was adjusted to 7.4 with $\mathrm{NaOH}$ ). The preheated extracellular solution was continuously perfused with similar setup compared to what is presented in Section 2.5.2. Patch pipettes (Harvard Apparatus Ltd., Holliston, MA, USA) were freshly prepared using PC-10 micropipette puller and then flame-polished with MF-830 microforge (both from Narishige Int., Tokyo, Japan).

APs were recorded in the gap-free mode in the current clamp from the spontaneously beating hiPSC-derived CMs. Current-clamp recordings were digitally sampled at $20 \mathrm{kHz}$ and filtered at $2 \mathrm{kHz}$ using low pass Bessel filter on recording amplifier. Beats per minute $(\mathrm{BPM}), \mathrm{AP}$ duration $\left(\mathrm{APD}_{50}\right.$ and $\mathrm{APD}_{90}$ ), $\mathrm{AP}$ amplitude (APA), and maximum diastolic potential (MDP) were analyzed from the recorded APs by using Origin 9.1 (OriginLab Corp., Northampton, USA). Only ventricular-like waveforms are presented here to avoid any biasness among different hiPSC lines. The ventricularlike CMs were characterized by $\mathrm{APD}_{90} / \mathrm{APD}_{50}<1.3$ and $\mathrm{APA}$ $>90 \mathrm{mV}$.

2.5.4. Real-Time qRT-PCR Analysis. After one week of culture, dissociated CMs were collected into a lysis solution buffer of CellsDirect One-Step qRT-PCR Kit (Life Technologies Ltd.) according to the manufacturer's instructions. Two replicate samples were collected and stored at $-70^{\circ} \mathrm{C}$ until the DNase I digestion and reverse transcription-specific target amplification (RT-STA) by using CellsDirect One-Step qRTPCR Kit. Real-Time qPCR was performed with Biomark HD system (Fluidigm Corp., San Francisco, USA) according to the manufacturer's instructions. The TaqMan assays (Life Technologies Ltd.) used in the qRT-PCR are collected in Table 1. All samples were analyzed in duplicate and the fold changes were calculated by the $2^{-\triangle \Delta C T}$ method [21]. EEF1A1 and GAPDH genes were used as endogenous control genes and UTA.04511.WT cell line was used as a calibrator.

2.5.5. Western Blot. hiPSC-derived CMs were lysed in MPER protein extraction reagent (Thermo Scientific, Life Technologies Ltd.), supplemented with complete protease inhibitor cocktail (Roche Diagnostics). The protein concentration was quantified with BCA protein assay kit (Thermo Scientific, Life Technologies Ltd.). $10 \mu \mathrm{g}$ of protein was run to $4-15 \%$ mini-PROTEAN TGX precast polyacrylamide gel 
(Bio-Rad, Hercules, CA, USA) and transferred to PVDF membrane (Amersham Hybond-P, GE Healthcare, Little Chalfont, UK). Membranes were blocked with 5\% milk for $1 \mathrm{~h}$ at RT and proteins were stained with MYBPC $(1: 1500$, sc166081, Santa Cruz Biotechnology), cTnT (1:2000, ab64623, Abcam), TPM1 (1:200, sc-73225, Santa Cruz Biotechnology), or $\beta$-actin (1:1000, sc-47778, Santa Cruz Biotechnology) primary antibodies over night at $+4^{\circ} \mathrm{C}$. Horseradish peroxidase(HRP-) conjugated polyclonal rabbit anti-mouse (DAKO, P0260) and rabbit anti-goat IgG (Santa Cruz Biotechnology, sc-2922) were used as secondary antibodies. Stained proteins were detected by using Clarity ECL substrate (Bio-Rad) and visualized by Molecular Imager ChemiDOc XRS+ (Bio-Rad). ImageJ software (National Institutes of Health, USA) was used to compare the expression of MYBPC, cTnT, and TPM1 with the $\beta$-actin expression from the same cell line.

2.6. Statistical Analysis. For statistical analysis, control cell lines and cell lines in each mutation were combined in groups: UTA.04602.WT and UTA.04511.WT hiPSC lines in WT-CM group, UTA.02912.HCMT and UTA.13602.HCMT in HCMTCM group, and UTA.07801.HCMM and UTA.06108.HCMM in HCMM-CM group. Mann-Whitney $U$ test with Bonferroni's correction was used to analyze the differences between WT-, HCMT-, and HCMM-CMs in cell size analysis, proportion of multinucleated CMs, and $\mathrm{Ca}^{2+}$ imaging experiments as well as in gene expression analysis. For the statistical comparison between the three groups, one-way ANOVA followed by Tukey test was used for the patch-clamp result analysis. $p<0.05$ was considered statistically significant. All error bars are presented as standard error of the mean (SEM).

\section{Results}

3.1. hiPSCs Were Derived from HCM Patients with Different Backgrounds. We derived hiPSCs from four patients carrying a HCM causing mutation either in TPM1 (TPM1-Asp175Asn) or in MYBPC3 (MYBPC3-Gln1061X). UTA.13602.HCMT and UTA.02912.HCMTs carry TPM1-Asp175Asn and UTA.07801. HCMM and UTA.06108.HCMM MYBPC3-Gln1061X mutation. The hiPSC lines and their mutations and abbreviations, used below, are presented in Table 2. UTA.13602.HCMT (46, $\mathrm{XX})$ is derived from a 48-year-old female, whose mother died suddenly at the age of 51. Our patient has had one collapse at the age of 20 with normal heart structure, but later slight thickening of septum $(16 \mathrm{~mm})$ has been observed. Currently she is not on medication due to low blood pressure. UTA.02912.HCMT (46, XY) is derived from a 33-year-old male whose family member has died suddenly at the age of 21. The maximal septal thickness of our patient has been measured to be $26 \mathrm{~mm}$ on echocardiogram. The patient has been asymptomatic but is currently on $\beta$-blocker medication. UTA.07801.HCMM (46, XY) is derived from a 61-year-old male with no SCDs in the family. On echocardiogram, his myocardial septum has been observed to be $25 \mathrm{~mm}$. He has atrial fibrillation and he is on $\beta$-blocker medication. Due to bradycardia and frequent nonsustained ventricular tachycardia episodes, an implantable cardioverter defibrillator (ICD)
TABLE 2: The hiPSC lines and their mutations and abbreviations used in the study.

\begin{tabular}{lccc}
\hline Group & Cell line & Mutation & $\begin{array}{c}\text { Name in } \\
\text { Figure 2(f) }\end{array}$ \\
\hline \multirow{2}{*}{ WT } & UTA.04602.WT & - & WT1 \\
& UTA.04511.WT & - & WT2 \\
\hline \multirow{2}{*}{ HCMT } & UTA.02912.HCMT & TPM1-Asp175Asn & HCMT1 \\
& UTA.13602.HCMT & TPM1-Asp175Asn & HCMT2 \\
\hline \multirow{2}{*}{ HCMM } & UTA.07801.HCMM & MYBPC3-Gln1061X & HCMM1 \\
& UTA.06108.HCMM & MYBPC3-Gln1061X & HCMM2 \\
\hline
\end{tabular}

has been implanted. UTA.06108.HCMM (46, XY) is derived from a 55-year-old male whose father died suddenly at the age of 36 and uncle at the age of 38. Our patient has been asymptomatic with maximal septal thickness of $22 \mathrm{~mm}$ on echocardiogram. He is not on medication due to low blood pressure. Control hiPSC lines were derived from healthy individuals: UTA.04602.WT (46, XX) from a 56-year-old female and UTA.04511.WT (46, XY) from a 34-year-old male.

The pluripotent characteristics of the hiPSC-lines used were assessed (Figure 1 and Supplementary Figures 1-5). UTA.04602.WT cell line has been characterized earlier [22]. All the lines formed colonies, which expressed proteins and genes typical for hPSCs. The virally transferred exogenous genes were silenced and karyotypes of the hiPSC lines were normal. The pluripotency of hiPSC lines was proven in vitro by $\mathrm{EB}$ formation or in vivo by teratoma formation. The presence of TPM1-Asp175Asn and MYBPC3-Gln1061X mutations in the patient-specific hiPSC lines was confirmed by custom TaqMan SNP Genotyping Assays (Supplementary Figure 6).

3.2. Mutation-Specific HCM Phenotypes Were Observed in hiPSC-Derived Cardiomyocytes. All the cell lines used in the present study differentiated into cardiomyocytes similarly. Beating aggregates were formed 14-20 days after the initiation of coculturing with END-2 cells and there was no difference between the lines when the beating areas appeared. After cardiac differentiation, beating clusters were dissociated into single cells and cultured for 1,3, and 6 weeks. The differences in the cell sizes and $\mathrm{Ca}^{2+}$ handling properties between two different mutations and control cells were analyzed in each time point. When comparing different types of CMs, HCMM-CMs were significantly larger than HCMT-CMs and WT-CMs in all three time points (Figures 2(a) and 2(b), $n=96-100, p<0.005$ in all cases). The enlargement of HCMT-CMs was detected after three weeks of culture when they were significantly larger than WT-CMs $(p<$ 0.005). Generally, the size of the hiPSC-derived CMs in all groups increased when the cells cultured for three weeks. Within each group, there were no differences in cell sizes between three and six weeks, except with HCMT-CMs, which seemed to be smaller in size 6-week time point. However, during 6 weeks of culture, cell types other than CMs had the tendency to overgrow the CM culture, which might have affected the cellular enlargement. This phenomenon was 

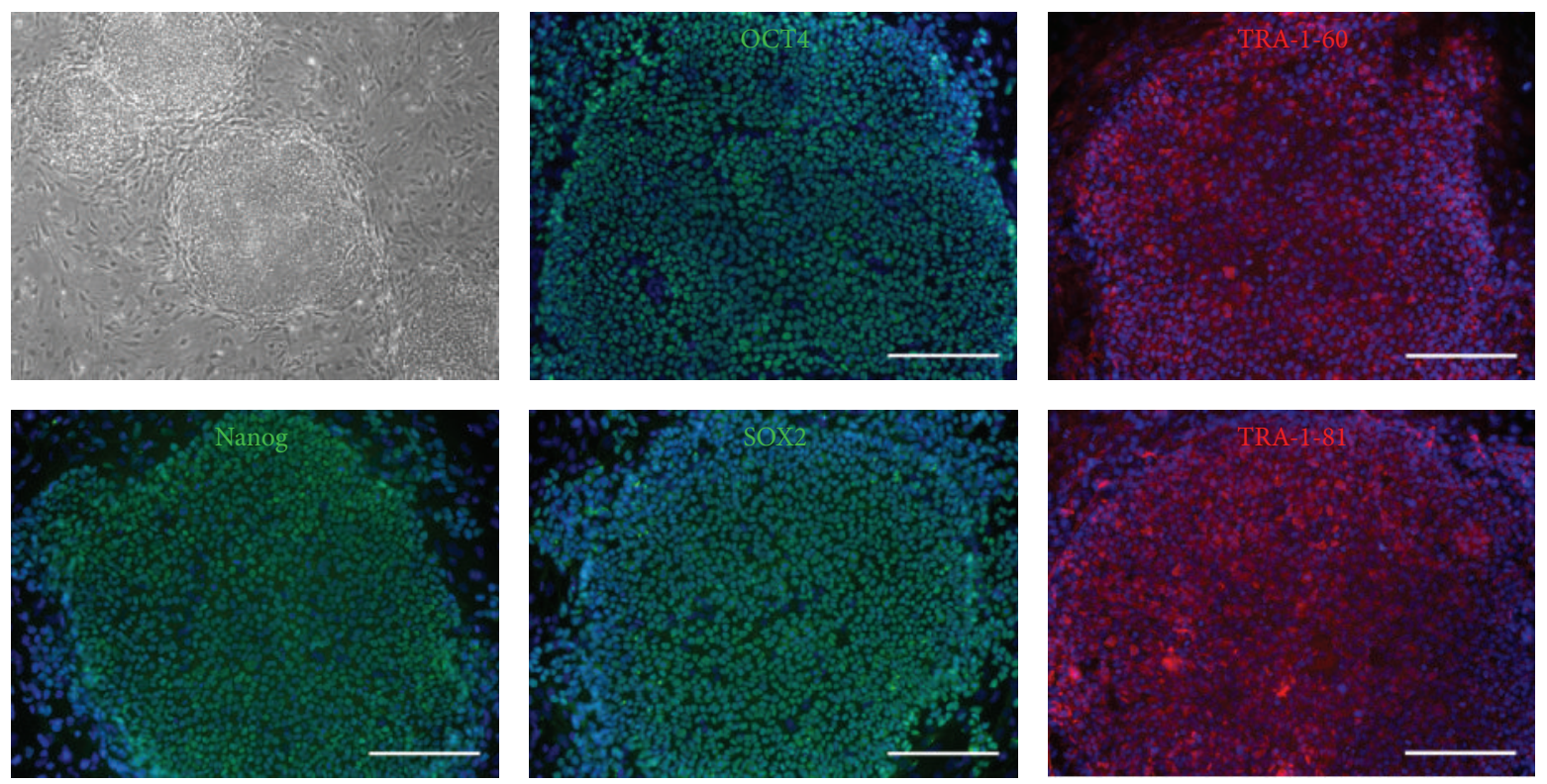

(a)

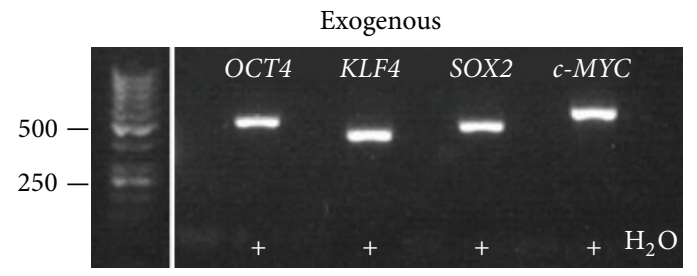

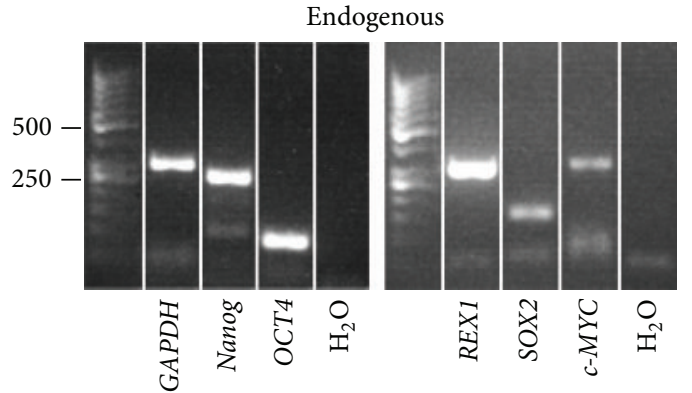

(b)

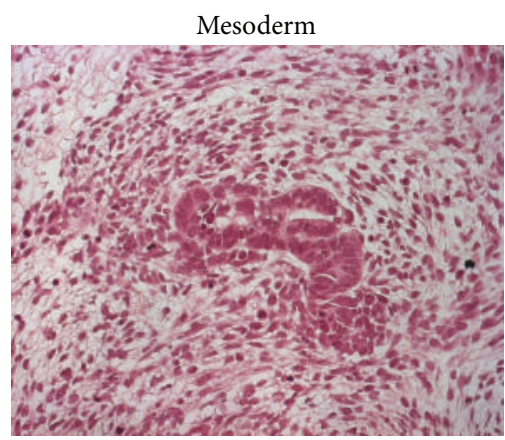

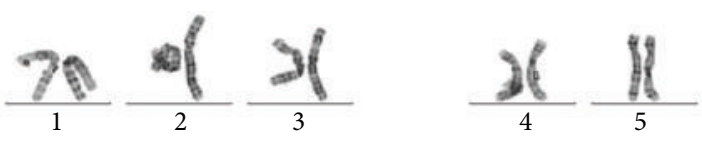

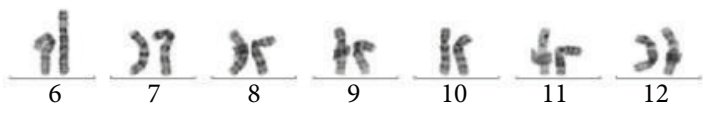

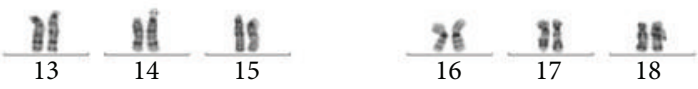$$
\frac{88}{19} \frac{88}{20} \quad \frac{36}{21} \frac{8 \pi}{22}
$$$$
\frac{d x}{X} \frac{}{Y}
$$

(c)
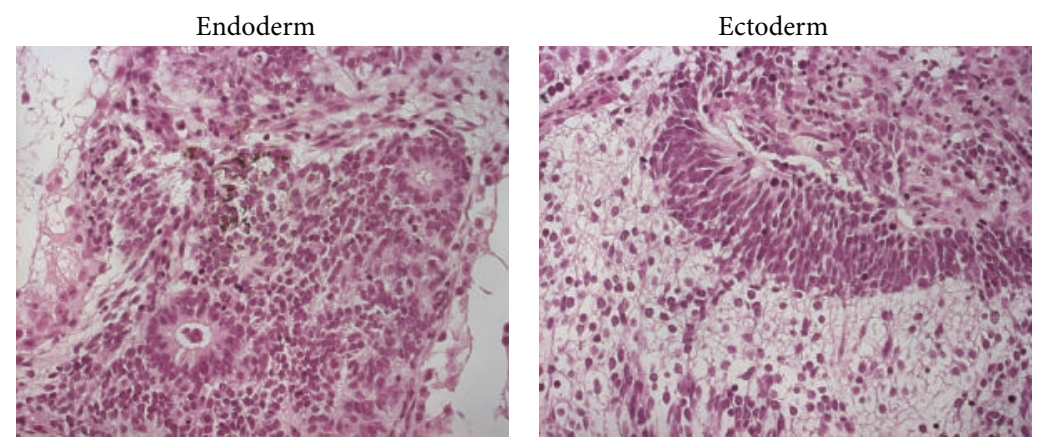

(d)

FIGURE 1: Characterization of UTA.13602.HCMT cell line. (a) The hiPSCs formed colonies expressing Nanog, OCT4, SOX2, TRA-1-60, and TRA-1-81. Scale bars: $200 \mu \mathrm{m}$. (b) The virally transferred Sendai exogenes, exo-OCT4 (483 bp), exo-KLF4 (410 bp), exo-SOX2 (451 bp), and exo$c$-MYC (532 bp), were silenced in the hiPSCs. + indicates positive controls, for which RNA was extracted from cells 1 week after transduction. hiPSCs expressed endogenous Nanog (287 bp), OCT4 (144 bp), REX1 (306 bp), SOX2 (151 bp), and c-MYC (328 bp). GAPDH (302 bp) was used as a housekeeping control. (c) The hiPSC line was karyotypically normal, 46 XX. (d) The pluripotency of hiPSCs was confirmed by in vivo teratoma assay, in which hiPSCs formed all three germ layers (mesoderm, endoderm, and ectoderm). 

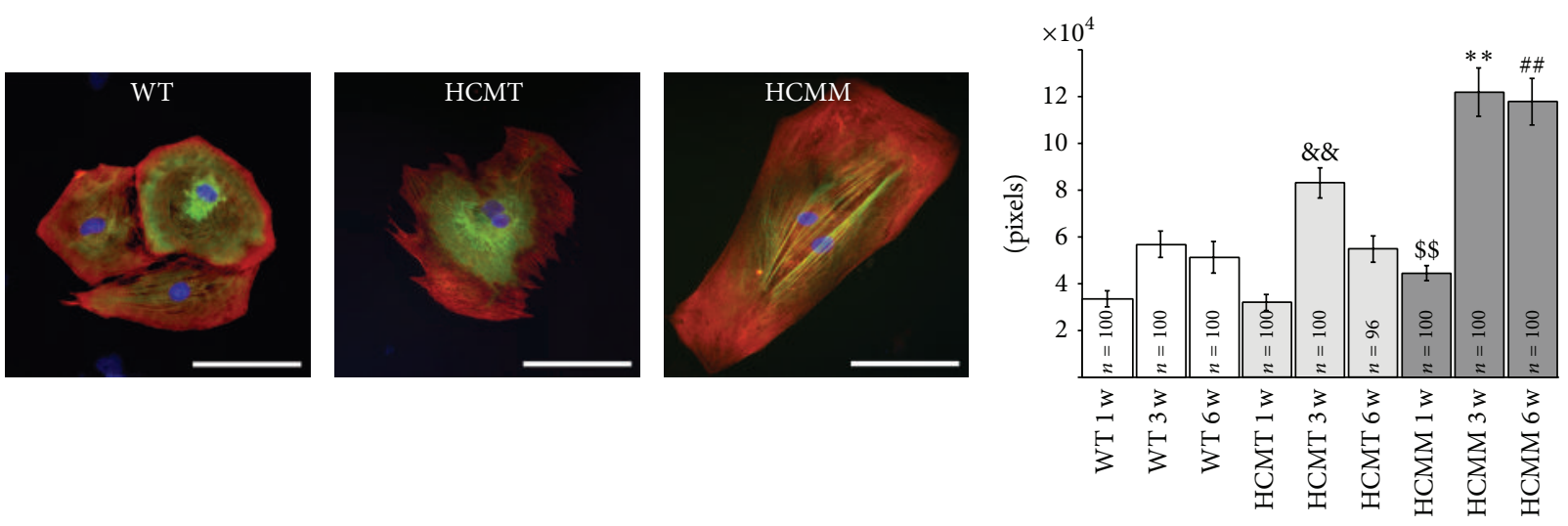

(a)

(b)
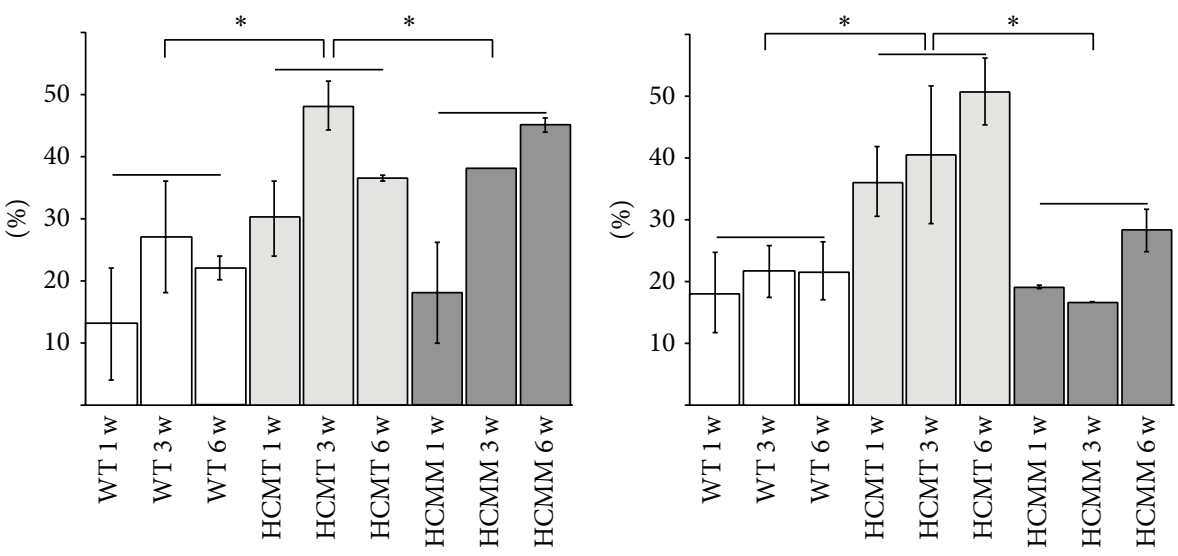

(c)

(d)
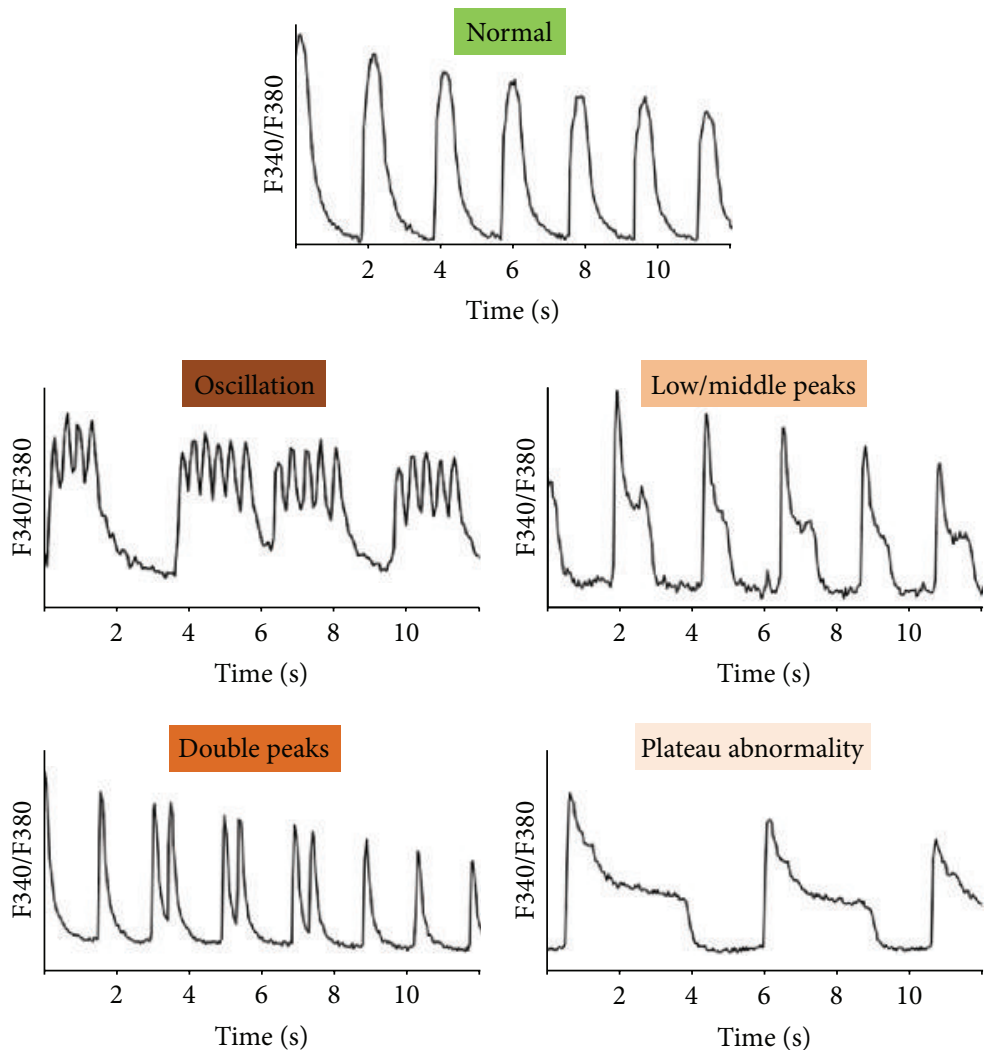

(e)

Figure 2: Continued. 


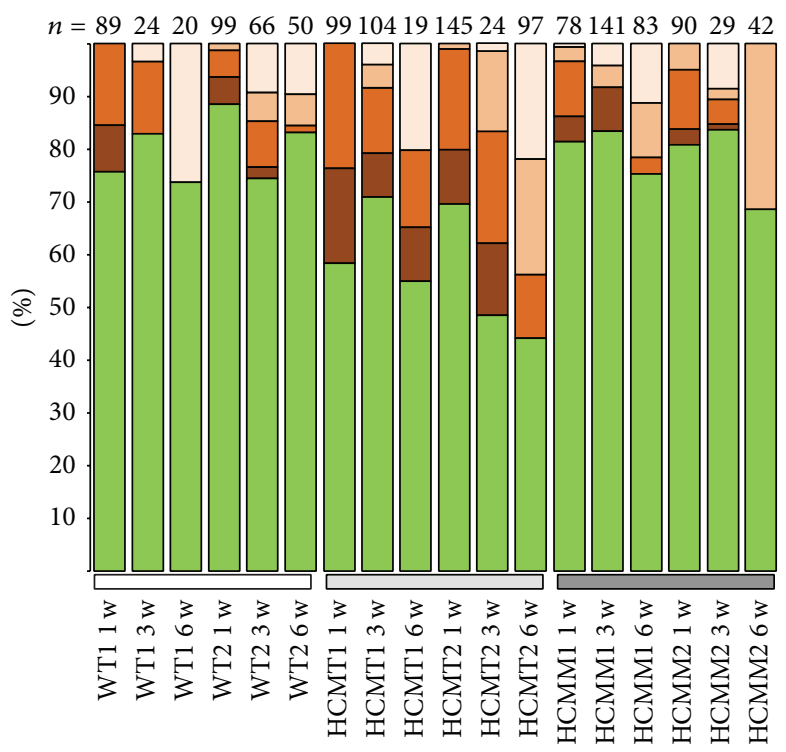

(f)

FIGURE 2: The cell size and $\mathrm{Ca}^{2+}$ handling of hiPSC-derived CMs after 1-, 3-, and 6-week culture as single cells. (a) Representative images of WT-CMs (WT), HCMT-CMs (HCMT), and HCMM-CMs (HCMM) stained with antibodies for cTnT (red) and MYBPC (green) proteins. Scale bars are $100 \mu \mathrm{m}$. (b) The size of the HCMM-CMs was significantly larger in all three time points when compared to WT- and HCMTCMs $\left({ }^{\$} p<0.005\right.$ when compared to WT-CMs or HCMT-CMs in the 1-week time point, ${ }^{* *} p<0.005$ when compared to WT-CMs or HCMT-CMs in 3-week time point, and ${ }^{\# \#} p<0.005$ when compared to WT-CMs or HCMT-CMs in 6-week time point). HCMT-CMs were significantly larger than WT-CMs in 3 -week time point $\left({ }^{\&} \& p<0.005\right.$ when compared to WT-CMs. $n=100$, except in HCMT $6 \mathrm{w}$ $n=96$.) (c) The proportion of the multinucleated CMs was significantly higher in HCMT-CMs than in WT-CMs and HCMM-CMs when both cell lines and all time points were combined for each group (in statistical analysis $n=6,{ }^{*} p<0.05$ ). The averages of multinucleated $\mathrm{CMs}$ were determined from the same cells, whose sizes and $n$ numbers are presented in (b). (d) Significantly more $\mathrm{CMs}$ with $\mathrm{Ca}^{2+}$ handling abnormalities were observed in HCMT-CMs than in WT-CMs and HCMM-CMs when both cell lines and all time points were combined for each group (in statistical analysis $n=6,{ }^{*} p<0.05$ ). The proportions of CMs with abnormalities in their $\mathrm{Ca}^{2+}$ handling were determined from the same $\mathrm{Ca}^{2+}$ imaging results presented in (f). The total $n$ numbers of the analyzed CMs are presented in (f). (e) Representative images of $\mathrm{Ca}^{2+}$ rhythm categories. (f) Distributions of hiPSC-derived CMs in different $\mathrm{Ca}^{2+}$ rhythm categories (e) in each time point. WT1 = UTA.04602.WT, WT2 = UTA.04511.WT, HCMT1 = UTA.02912.HCMT, HCMT2 = UTA.13602.HCMT, HCMM1 = UTA.07801.HCMM, and HCMM2 = UTA.06108.HCMM.

observed especially with UTA.13602.HCMT cell line (data not shown). When all time points in each group (WT-CM, HCMT-CM, and HCMM-CM) were combined, the number of multinucleated CMs was significantly higher in HCMTCMs than in WT-CMs and HCMM-CMs $(n=6$ in statistical analysis, $p<0.05$, Figure 2(c)).

The $\mathrm{Ca}^{2+}$ handling properties of hiPSC-derived CMs were studied by $\mathrm{Ca}^{2+}$ imaging. HCMT-CMs had significantly more abnormalities than WT-CMs and HCMM-CMs, when all time points in each group were combined (Figure $2(\mathrm{~d}), n=$ 6 in statistical analysis, $p<0.05$ ). Spontaneously beating CMs were divided into five different rhythm categories (normal, oscillation, low/middle peaks, double peaks, and plateau abnormality) based on the abnormalities observed in their $\mathrm{Ca}^{2+}$ signals (Figures 2(e) and 2(f)). HCMT-CMs had significantly higher number of double peaks than HCMMCMs ( $p<0.05, n=6$ in statistical analysis) when both cell lines and all time points were combined for each group.

3.3. Action Potential Characteristics of WT and HCM hiPSCDerived Cardiomyocytes. The spontaneous action potentials were recorded from the beating hiPSC-derived CMs to establish the electrophysiological baselines. Most of the cells $(>80 \%)$ were ventricular-like CMs in all the hiPSC lines studied. For this reason, only ventricular-like waveforms are presented here. We first analyzed the percentage of the arrhythmias in each cell line (Figures 3(a)-3(f)) and found similar percentage in both cell lines within the groups (UTA.04602.WT (13\%) versus UTA.04511.WT (15\%), UTA.02912.HCMT (42\%) versus UTA.13602.HCMT (47\%), and UTA.07801.HCMM (50\%) versus UTA.06108.HCMM $(50 \%))$. Based on the percentage of the arrhythmias, we combined hiPSC-derived CMs into groups (WT-CM, HCMT$\mathrm{CM}$, and HCMM-CM) for further analysis.

Both HCMT-CMs and HCMM-CMs had more arrhythmic events including delayed after depolarizations (DADs) and early after depolarizations (EADs) when compared to the WT-CMs (WT-CM (14\%), HCMTCM (45\%), and HCMM-CM (50\%)). We quantified the occurrence of DADs in hiPSC-derived CMs as a rate (DADs/min) calculated as total number of DADs/total number of APs. We found that the DAD rate in HCMMCMs was significantly higher than in WT-CMs (Figure 3(g), $p<0.005)$. 


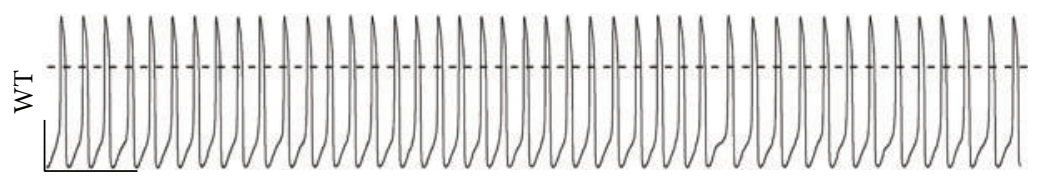

(a)

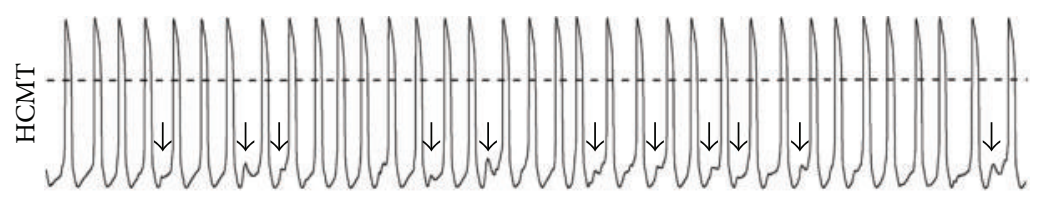

(b)

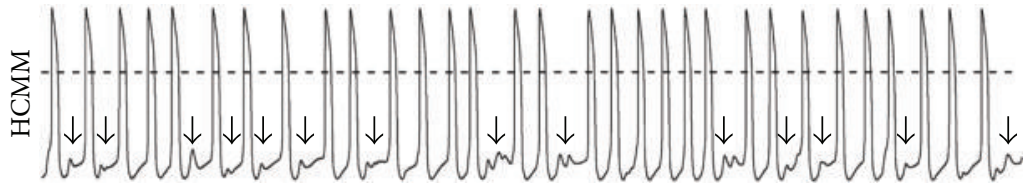

(c)

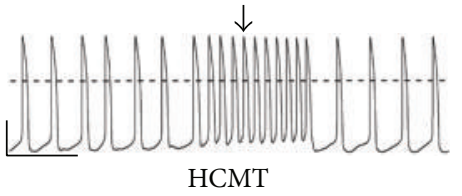

(d)
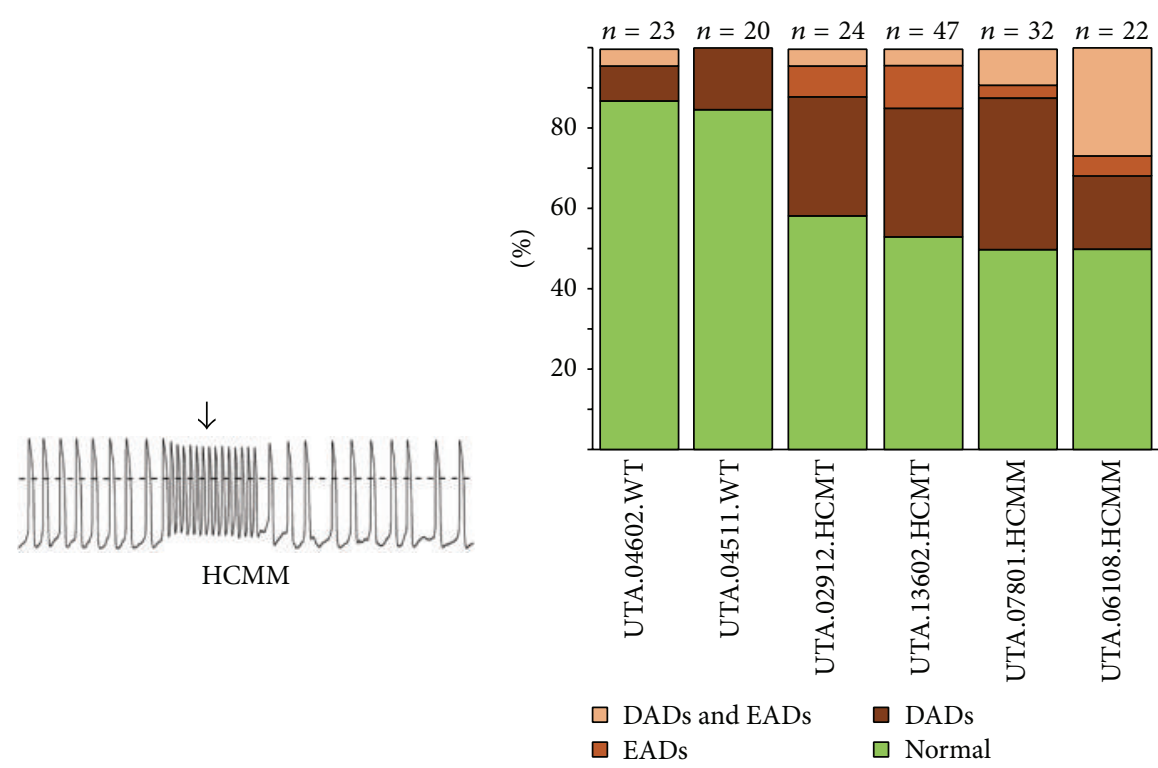

(e)

(f)

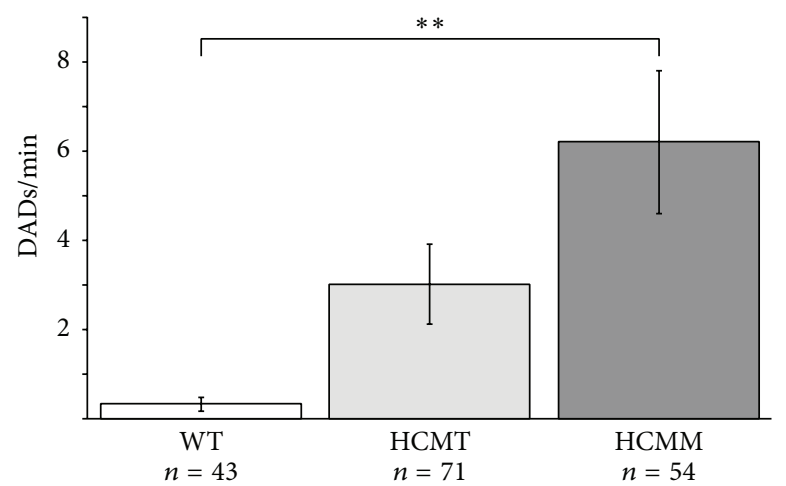

(g)

FIGURE 3: Arrhythmogenic events (DADs and EADs) were observed in HCM-CMs. (a)-(e) Representative recordings of control hiPSCderived CMs (WT) and hiPSC-derived CMs carrying TPM1-Asp175Asn (HCMT) or MYBPC3-Gln1061X (HCMM) mutations. Typical DADs (arrows) are presented in (b) and (c) and EADs (arrows) in (d) and (e) for HCMT-CMs and HCMM-CMs, respectively. Scale bars represent $40 \mathrm{mv}$ and 5 seconds, respectively. Scale bars in (a) are representative for (b) and (c), and scale bars in (d) are representative for (e). (f) Distribution of CMs exhibiting arrhythmogenic events in each cell line. (g) DAD rate was significantly higher in HCMM-CMs than in WTCMs $\left({ }^{* *} p<0.005\right)$. 
TABLE 3: AP properties of ventricular-like CMs derived from control hiPSC lines (WT) and from hiPSC lines carrying TPM1-Asp175Asn (HCMT) or MYBPC3-Gln1061X (HCMM) mutations. In the results, the data of each group is comprised from two separate cell lines.

\begin{tabular}{|c|c|c|c|c|c|c|}
\hline Group & $n$ & $\begin{array}{c}\text { Beating rate } \\
(\mathrm{BPM})\end{array}$ & $\begin{array}{c}\mathrm{APD}_{50} \\
(\mathrm{~ms})\end{array}$ & $\begin{array}{c}\mathrm{APD}_{90} \\
(\mathrm{~ms})\end{array}$ & $\begin{array}{l}\mathrm{APA} \\
(\mathrm{mV})\end{array}$ & $\begin{array}{l}\mathrm{MDP} \\
(\mathrm{mV})\end{array}$ \\
\hline $\mathrm{WT}$ & 43 & $58.1 \pm 2.3$ & $277.3 \pm 13.0$ & $323.6 \pm 13.9$ & $119.5 \pm 1.1$ & $-76.8 \pm 0.8$ \\
\hline HCMT & 71 & $48.4 \pm 1.5^{* *}$ & $372.3 \pm 13.2^{* *}$ & $433.1 \pm 14.0^{* *}$ & $121.2 \pm 1.1$ & $-75.8 \pm 0.7$ \\
\hline HCMM & 54 & $47.1 \pm 1.8^{* *}$ & $319.5 \pm 13.7^{\$}$ & $377.6 \pm 15.0^{*, \$}$ & $124.3 \pm 1.4^{*}$ & $-77.9 \pm 0.8$ \\
\hline
\end{tabular}

${ }^{*}$ HCMT or HCMM versus WT.

${ }_{\$} \mathrm{HCMM}$ versus HCMT.

$\$$ or $^{*} p<0.05$ and ${ }^{* *} p<0.005$.

The average $\mathrm{APD}$ at $50 \%$ repolarization $\left(\mathrm{APD}_{50}\right)$ and $90 \%$ repolarization $\left(\mathrm{APD}_{90}\right)$ of $\mathrm{HCMT}-\mathrm{CM}$ s was significantly longer than those of the WT-CMs $\left(\mathrm{APD}_{50}(p<0.005)\right.$ and $\left.\mathrm{APD}_{90}(p<0.005)\right)$ and HCMM-CMs $\left(\mathrm{APD}_{50}(p<0.05)\right.$ and $\mathrm{APD}_{90}(p<0.05)$ ) (Table 3$) . \mathrm{APD}_{90}$ of HCMM-CMs was significantly longer than that of the WT-CMs $(p<0.05)$ (Table 3). Furthermore, the beating rates of both HCMTCMs and HCMM-CMs were significantly lower than in WTCMs (WT-CM versus HCMT-CM $(p<0.005)$ and WT-CM versus HCMM-CM $(p<0.005))$. In addition, the APA of HCMM-CMs was significantly higher than in the WT-CMs $(p<0.05)$. However, no significant differences were found for the MDP between any groups (Table 3 ).

3.4. Differences in the Gene Expression Profiles of hiPSCDerived Cardiomyocytes. Dissociated hiPSC-derived CMs were cultured for one week before qRT-PCR analysis was performed. The results are presented in Figure 4 . The expression of sarcomeric genes MYBPC3, TNNT2, ACTN2, TTN, MYL7, and MYL9 was significantly higher in both HCMTCMs and HCMM-CMs than in the WT-CMs $(p<0.005$ in all cases). The expression of TPM1 and TNNC1 was significantly increased only in the HCMM-CMs when compared to WTCMs ( $p<0.005$ in both cases). On the other hand, the expression of MYH6 was on the same level in all hiPSCderived CMs. Moreover, the expression of some sarcomeric genes (TNNT2, ACTN2, TNNC1, TTN, MYL7, and MYL9) was significantly higher in the HCMM-CMs than in the HCMT-CMs $(p<0.005$ in other than TNNT2 and MYL9 $p<0.05)$. The expression of natriuretic peptide A (NPPA) was similar in all hiPSC-derived CMs while the expression of natriuretic peptide $\mathrm{B}(N P P B)$ was increased in HCMT-CMs and HCMM-CMs when compared to WT-CMs $(p<0.005$ in both cases). Nodal marker HCN4 was also significantly increased in both HCM-CMs when compared to WT-CMs $(p<0.05$ for HCMT-CMs versus WT-CMs and $p<0.005$ for HCMM-CMs versus WT-CMs). Further, the highest expression of potassium channel KCNQ1 and sodium channel SCN5A as well as sodium calcium exchanger SLC8A1 was observed in HCMM-CMs $(p<0.005$ when compared to WT-CMs in all cases).

We found differences also in the expression of genes related to the $\mathrm{Ca}^{2+}$ handling. The expression of CACNA1C and PLN was increased in both HCMT-CMs $(p<0.05$ for CACNA1C and $p<0.005$ for PLN) and HCMM-CMs ( $p<0.005$ in both cases) when compared to WT-CMs while the expression of ATP2A2 and ITPR2 was on the same level in all hiPSC-derived CMs. However, the expression of CASQ2 and RYR2 was significantly higher in HCMT-CMs and HCMM-CMs when compared to WT-CMs $(p<0.005$ in all cases). Moreover, the expression of RYR2 was almost six times higher in the HCMM-CMs than in the WT-CMs $(p<0.005)$ and around three times higher than in HCMTCMs $(p<0.005)$.

3.5. Truncated MYBPC Protein Was Not Detected in hiPSCDerived Cardiomyocytes Carrying MYBPC3-Gln1061X Mutation. Both wild type and the mutant TPM1 mRNA were present in HCM-CMs carrying the TPM1-Asp175Asn mutation while hiPSC-derived control CMs expressed only wild type mRNA. However, in hiPSC-CMs carrying the MYBPC3Gln1061X mutation the mutant mRNA was not clearly detected (Supplementary Figure 7). At a protein level all hiPSC-derived CMs expressed MYBPC, cTnT, and TPM1 (Figures 5(a) and 5(b)). The truncated MYBPC protein (predicted size: $116 \mathrm{kDa}$ ) was not detected in HCMM-CMs with western blot analysis (Figure 5(b)). However, the expression level of total MYBPC was slightly reduced in HCMM-CMs when compared to WT-CMs (Figure 5(c)). In addition, the expression of cTnT and TPM1 was elevated in both HCMCMs (Figure 5(c)).

\section{Discussion}

Here we have analyzed characteristics of hiPSC-derived HCM-CMs carrying either TPM1-Asp175Asn or MYBPC3$G \ln 1061 X$ gene mutation. The size of the MYBPC3-Gln1061X CMs was significantly larger than that of TPM1-Asp175Asn CMs, while the CMs carrying the latter mutation had significantly more abnormal $\mathrm{Ca}^{2+}$ transients. Additionally, CMs with TPM1-Asp175Asn mutation had significantly more prolonged action potentials. However, both types of HCMCMs had increased amount of arrhythmogenic events (DADs and EADs) in electrophysiological recordings when compared to control CMs. In addition to morphological and functional differences, also gene expression profiles were different between CMs carrying either TPM1-Asp175Asn or MYBPC3-Gln1061X gene mutation.

Genetic HCM is primarily due to mutations in sarcomeric genes, while changes at the cellular level include disturbed $\mathrm{Ca}^{2+}$ metabolism and decreased contraction force generation in addition to enlarged cell size [23]. To our knowledge, 

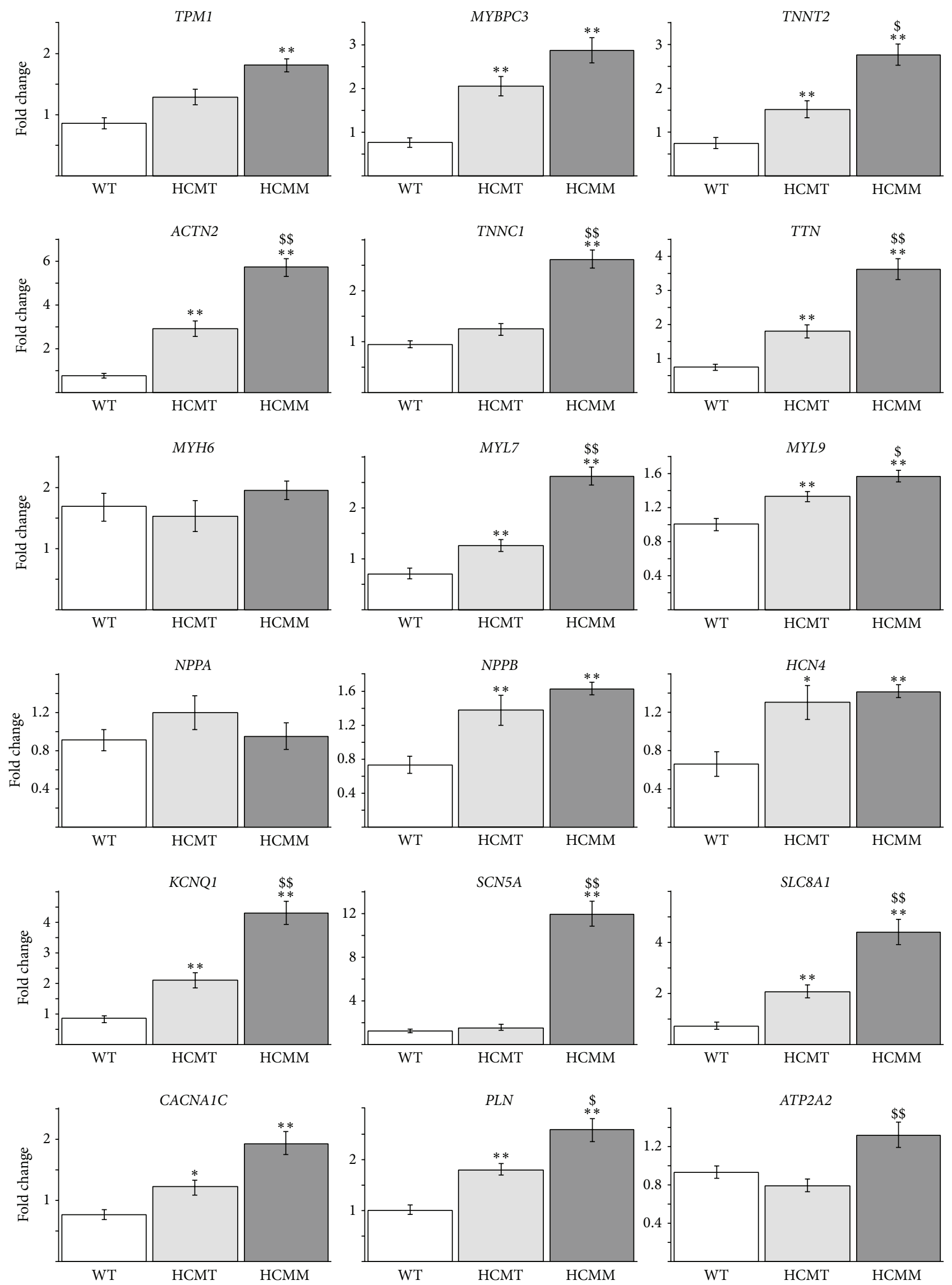

Figure 4: Continued. 

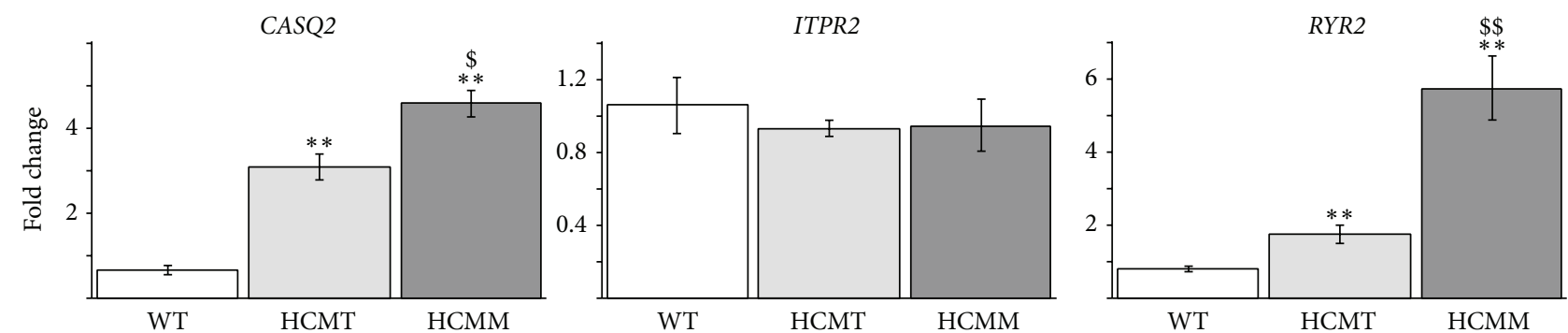

FIGURE 4: Gene expression profiles in control hiPSC-derived CMs (WT) and in hiPSC-derived CMs carrying TPM1-Asp175Asn (HCMT) or MYBPC3-Gln1061X (HCMM) mutations. ( ${ }^{*}$ represents HCMT or HCMM versus WT, and ${ }^{\$}$ represents HCMT versus HCMM. ${ }^{* *}$ or $\$ p<0.005,{ }^{*}$ or $\$ p<0.05 n=16$, except for NPPA $\left.n=8\right)$.

total of three reports studying the characteristics as well as the pathophysiological mechanisms of the HCM by using the patient-specific hiPSCs have been published [12-14]. In two of these publications, the mutation is located in the MYH7 (MYH7-R663H or MYH7-R442G) [12, 13], whereas, in the most recent publication, the hiPSCs were derived from three HCM patients, from whom one carried the MYBPC3999-1004del2, while the other mutations were unknown [14]. In addition to these three publications, hiPSC-derived CMs carrying MYBPC3 mutations have been used in one study where the effects of serum on the phenotype of neonatal rat CMs as well as hPSC-derived CMs have been explored [24]. hiPSC-derived CMs carrying MYBPC3 mutation were used only when studying the effects of serum on the cellular enlargement [24]. In this current study, we obtained hiPSCs from HCM patients carrying either the MYBPC3-Gln1061X or TPM1-Asp175Asn mutation. We believe that this is the first report where hiPSC-derived CMs carrying different gene mutations have been compared in the same study with similar experimental settings.

Our HCM-CMs demonstrated cellular enlargement similarly to previous HCM studies with hiPSCs [12-14]. However, we observed a significant difference in the cellular enlargement between the two HCM mutations. CMs carrying the MYBPC3-Gln1061X mutation presented more pronounced and earlier cellular enlargement than CMs carrying the TPM1-Asp175Asn mutation. In a previous study, serum has been shown to mask hypertrophic phenotype of the CMs with mutations in the MYBPC3 [24]. The CMs from the HCM patients were larger in serum-free conditions without any external stimuli, while the serum seemed to increase the cellular enlargement in WT-CMs but not in CMs with HCM mutations [24]. We used $20 \%$ serum in our CM culture medium that did not seem to mask the cellular enlargement with our CMs. Already after one week of culture, CMs carrying MYBPC3-Gln1061X mutation were significantly larger than the WT-CMs. The enlargement of CMs carrying TPM1Asp175Asn mutation was detectable only after three weeks of culture. In the previous clinical studies, as well as in the patient data analyzed in this current study, the hypertrophy has been in the same range in patients carrying either of these two mutations [2]. Therefore, these differences in CM size between the two mutations do not correlate with the extent of clinical hypertrophy.
The higher $\mathrm{Ca}^{2+}$ sensitivity, observed in animal models and myectomy samples, has been suggested to be a common feature for all HCM mutations $[25,26]$. In the previous studies with hiPSC-derived CMs, irregularities in $\mathrm{Ca}^{2+}$ transients have been observed in the MYH7-R663H and the MYH7$R 442 G$ mutations $[12,13]$. The higher $\mathrm{Ca}^{2+}$ sensitivity has been related to lower phosphorylation levels of the MYBPC and the Troponin I proteins and the difference could at least partly be explained by hypophosphorylation of the sarcomeric proteins compared to the actual mutations [26]. In our study, the amount of abnormalities in $\mathrm{Ca}^{2+}$ transients was significantly increased only in the hiPSC-derived CMs with the TPM1-Asp175Asn mutation. Indeed, the amount of irregularities in $\mathrm{Ca}^{2+}$ handling properties was similar in the MYBPC3-Gln1061X compared to that in the WT-CMs. The phosphorylation of the proteins was not analyzed in this current study. However, we analyzed the expression of genes related to $\mathrm{Ca}^{2+}$ handling and they were at the highest level in hiPSC-derived CMs carrying the MYBPC3-Gln1061X mutation. This might be at least partly due to the larger cell size of these CMs. Han and coworkers found decreased level of RYR2 expression in HCM-CMs carrying MYH7-R442G mutation [13], while in our study the expression of $R Y R 2$ was significantly higher in both mutations and almost six times higher in the hiPSC-derived CMs carrying the MYBPC3Gln1061X mutation than in the WT-CMs. These observations suggest that abnormal $\mathrm{Ca}^{2+}$ transients in HCM-CMs carrying different mutations may be caused by distinct mechanisms.

One of the fundamental features of the HCM is its association with ventricular arrhythmias responsible for severe cardiac malfunctions including sudden cardiac death $[27,28]$. We found increased amount of arrhythmogenic events (DADs and EADs) in both HCM-CMs. Furthermore, decreased beating rate was observed in both types of HCMCMs, which could be due to higher occurrence of DADs between the two consecutive APs. In addition, the $\mathrm{APD}_{90}$ of hiPSC-derived CMs carrying either TPM1-Asp175Asn or MYBPC3-Gln1061X mutation was longer than in WT-CMs, which is in line with previous findings with different mutations $[12,13,29]$. The mechanism of arrhythmias in HCM is not yet fully understood; however, imbalances in $\mathrm{Ca}^{2+}$ homeostasis are considered as a main cause of arrhythmias shown in the previous studies [12, 13]. Clinically, patients carrying TPM1-Asp175Asn mutation have been reported to 

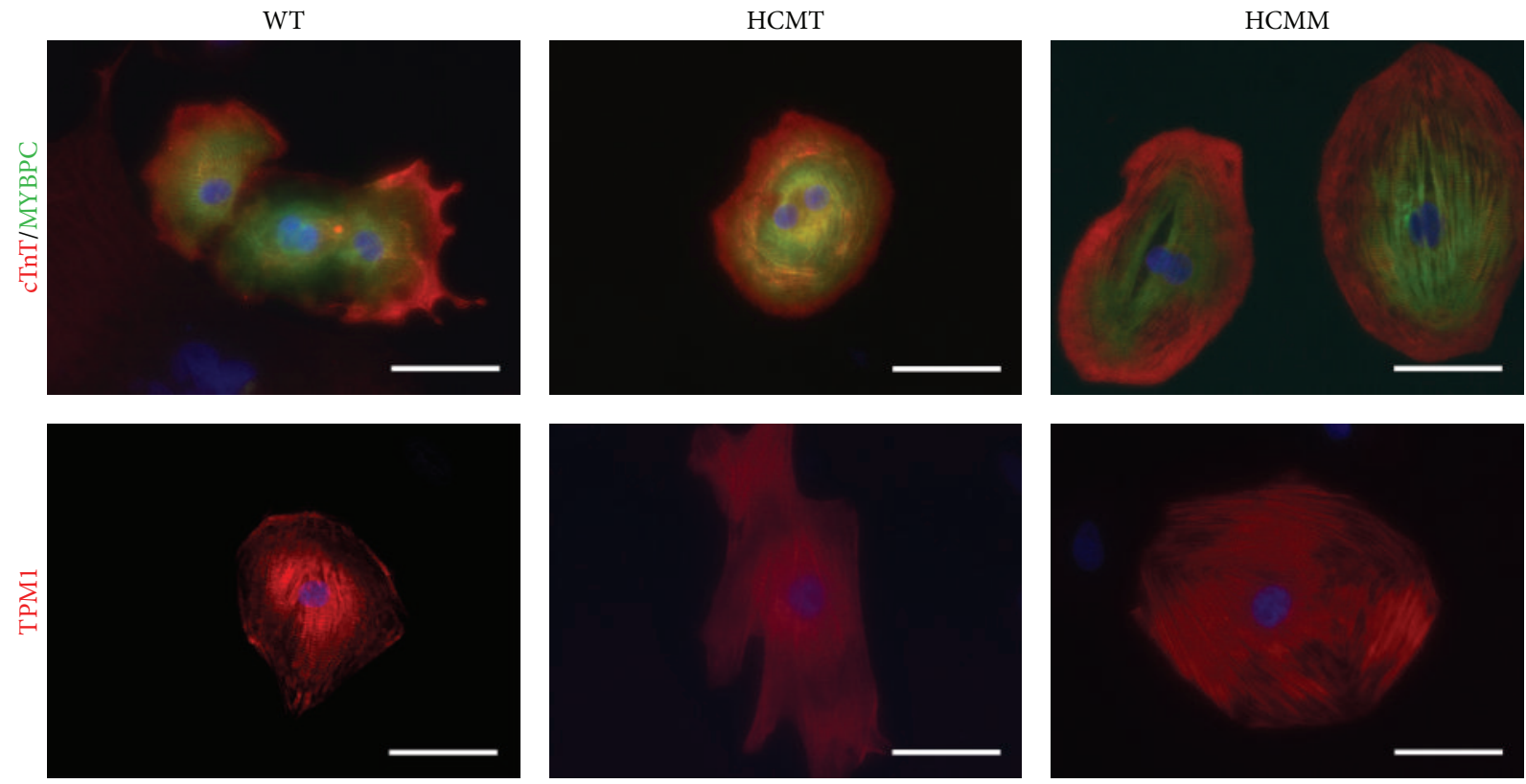

(a)
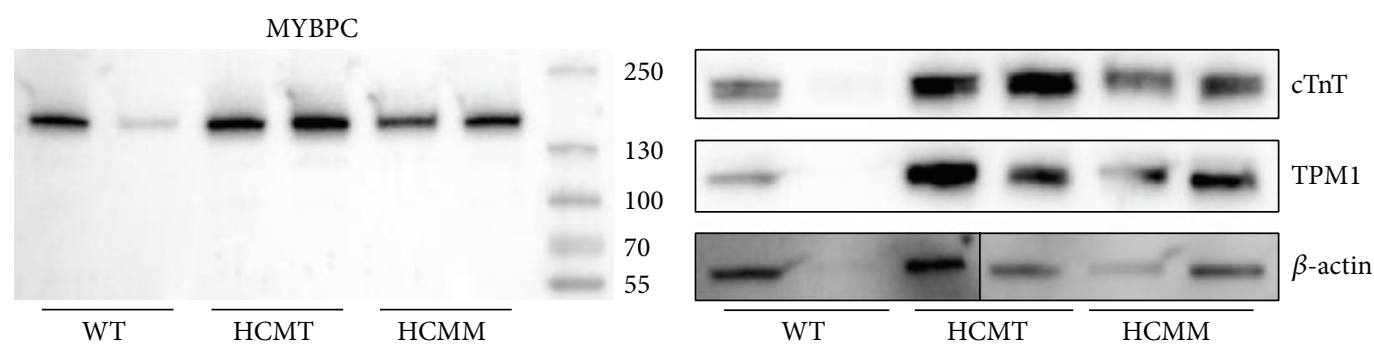

(b)
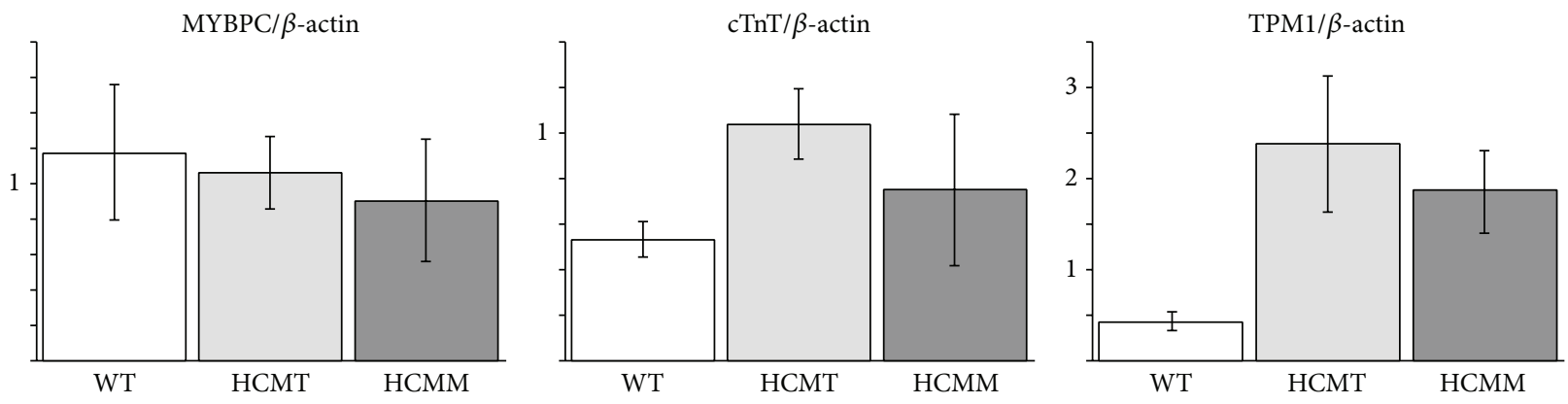

(c)

FIgure 5: Cardiac-specific protein expression in hiPSC-derived CMs. (a) Representative images of hiPSC-derived CMs carrying TPM1Asp175Asn mutation (HCMT) or MYBPC3-Gln1061X mutation (HCMM) and hiPSC-derived control CMs (WT) stained with cTnT, MYBPC, and TPM1. These images are not to quantify the protein expression but to demonstrate the presence of cTnT, MYBPC, and TPM1 proteins in the hiPSC-derived CMs. (b) hiPSC-derived CMs from all cell lines expressed MYBPC, cTnT, and TPM1 proteins. The truncated MYBPC was not detected in HCMM cells (size of the wild type protein $141 \mathrm{kDa}$ and the predicted size of the truncated protein $117 \mathrm{kDa}$ ). (c) The expression of MYBPC, cTnT, and TPM1 in WT-CMs, HCMT-CMs, and HCMM-CMs normalized to the expression of $\beta$-actin. Protein expressions were quantified from western blots using ImageJ software. Quantitation data show the averages of MYBPC/ $\beta$-actin, cTnT/ $\beta$-actin, and TPM1/ $\beta$ actin relations from hiPSC-derived CMs from two different hiPSC lines in each group. Because of the lack of replicates, statistical analysis was not performed. 
be more prone to arrhythmias than those carrying MYBPC3Gln1061X mutation [30]. Our data with hiPSC-derived CMs support this finding by demonstrating more abnormal $\mathrm{Ca}^{2+}$ transients and longer $\mathrm{APD}_{90}$ in TPM1-Asp175Asn CMs than in HCM-CMs carrying the MYBPC3-Gln1061X mutation.

Like most of the HCM mutations located in the MYBPC3, also $M Y B P C 3-G \ln 1061 X$ is a nonsense mutation that leads to premature stop-codon [31]. Nonsense mutations are suggested to act through haploinsufficiency in which the mutated protein is either degraded or not produced at all. The truncated form of MYBPC has not been found in human cardiac samples while the total expression level of MYBPC has been reported to vary from being decreased to even increased [32-36]. Interestingly, when studying myectomy samples from HCM hearts with MYBPC3 mutation, Helms et al. observed that the total amount of $M Y B P C 3$ mRNA was increased, while the total amount of MYBPC protein was on the same level compared to that in the control hearts. They hypothesized that the upregulation of the MYBPC3 compensates the degraded truncated MYBPC protein [35]. We could not detect mutant allele on mRNA expression level or truncated MYBPC protein in hiPSC-derived CMs carrying the $M Y B P C 3-G \ln 1061 X$ mutation. Similar absence of truncated protein has been reported earlier with a different MYBPC mutation in hiPSC-derived CMs [14]. These data suggest that the mutant mRNA might be degraded. However, further research is still needed to confirm the results and to discover the actual degradation mechanism.

The two HCM mutations analyzed in this study are the most frequent mutations in Finland accounting for about $18 \%$ of all Finnish HCM patients [3]. In our study, we found differences in the morphological and biochemical properties, as well as in $\mathrm{Ca}^{2+}$ cycling and electrophysiological properties between the CMs carrying either TPM1Asp175Asn or MYBPC3-Gln1061X mutation. However, we have not analyzed the possible effects of other gene mutations and epigenetic factors on the phenotype differences observed in our study. Additionally, we used only two hiPSC lines from two different patients in each mutation in our experiments. In the future, we need to extend our studies including further patients and studying the effects of additional gene mutations and epigenetic factors. The immature nature of hiPSCderived CMs is a general limitation when using these cells in disease modeling. Further development in the differentiation and maturation protocols will increase the reliability of studies conducted with hiPSC-derived CMs. Finally, the exact pathophysiology in abnormal $\mathrm{Ca}^{2+}$ transients or electrical abnormalities is not known and further research with combined patch clamp and $\mathrm{Ca}^{2+}$ imaging is required in the future to reveal the significance of the cellular findings for clinical phenotypes as well as for treatment options.

\section{Conclusions}

In conclusion, both HCM hiPSC-derived CMs either carrying the TPM1-Asp175Asn or MYBPC3-Gln1061X mutation exhibited pathological changes related to HCM. However, significant differences between the two mutations were observed. The hiPSC-derived cell models, established in this study, can be exploited to study further the pathophysiological mechanisms of HCM as well as to screen drugs and potentially optimize treatments in mutation-specific way.

\section{Abbreviations}

\begin{tabular}{|c|c|}
\hline AP: & Action potential \\
\hline APA: & Action potential amplitude \\
\hline APD: & Action potential duration \\
\hline bFGF: & Basic fibroblast growth factor \\
\hline BPM: & Beats per minute \\
\hline CM: & Cardiomyocyte \\
\hline CPVT: & $\begin{array}{l}\text { Catecholaminergic polymorphic } \\
\text { ventricular tachycardia }\end{array}$ \\
\hline cTnT: & Troponin $\mathrm{T}$ \\
\hline DAD: & Delayed after depolarization \\
\hline DAPI: & 40,6-Diamidino-2-phenylindole \\
\hline DCM: & Dilated cardiomyopathy \\
\hline EAD: & Early after depolarization \\
\hline EB: & Embryoid body \\
\hline END-2: & Mouse endodermal-like cells \\
\hline FBS: & Fetal bovine serum \\
\hline HCM: & Hypertrophic cardiomyopathy \\
\hline HCM-CM: & $\begin{array}{l}\text { Cardiomyocytes derived from hiPSCs } \\
\text { carrying HCM mutation }\end{array}$ \\
\hline HCMM-CM: & $\begin{array}{l}\text { Cardiomyocytes derived from hiPSCs } \\
\text { carrying MYBPC3-Gln1061X mutation }\end{array}$ \\
\hline HCMT-CM: & $\begin{array}{l}\text { Cardiomyocytes derived from hiPSCs } \\
\text { carrying TPM1-Asp175Asn mutation }\end{array}$ \\
\hline hiPSC: & Human induced pluripotent stem cell \\
\hline hPSC: & Human pluripotent stem cell \\
\hline HRP: & Horseradish peroxidase \\
\hline ICD: & Implantable cardioverter defibrillator \\
\hline ko: & Knockout \\
\hline ko-SR: & Knockout serum replacement \\
\hline MDP: & Maximum diastolic potential \\
\hline MEF: & Mouse embryonic fibroblast \\
\hline MYBPC: & Myosin-binding protein $\mathrm{C}$ \\
\hline MYH7: & $\beta$-myosin heavy chain \\
\hline NEAA: & Nonessential amino acids \\
\hline RT-STA: & $\begin{array}{l}\text { Reverse transcription-specific target } \\
\text { amplification }\end{array}$ \\
\hline SCD: & Sudden cardiac death \\
\hline SEM: & Standard error of the mean \\
\hline TPM1: & $\alpha$-tropomyosin \\
\hline WT-CM: & Cardiomyocytes derived from control \\
\hline
\end{tabular}

\section{Conflict of Interests}

The authors declare that there is no conflict of interests regarding the publication of this paper.

\section{Acknowledgments}

This study was funded by the Finnish Foundation for Cardiovascular Research, the Finnish Funding Agency for 
Technology and Innovation, Competitive Research Funding of the Pirkanmaa Hospital District, Finnish Cultural Foundation, Aarne and Aili Turunen Foundation, Maud Kuistila Memorial Foundation, and Aarne Koskelo Foundation. The authors would like to acknowledge Markus Haponen, Merja Lehtinen, Kirsi Penttinen, Julia Silvennoinen, and Henna Venäläinen for the general technical support, Turkka Mannila for the help with data analysis, Reija Autio for the help with statistical analysis, and Christine Mummery (Leiden University Medical Center, Leiden, Netherlands) for providing the END-2 cells.

\section{References}

[1] B. J. Maron, S. R. Ommen, C. Semsarian, P. Spirito, I. Olivotto, and M. S. Maron, "Hypertrophic cardiomyopathy: present and future, with translation into contemporary cardiovascular medicine," Journal of the American College of Cardiology, vol. 64, no. 1, pp. 89-99, 2014.

[2] P. Jääskeläinen, T. Heliö, K. Aalto-Setälä et al., "A new common mutation in the cardiac beta-myosin heavy chain gene in Finnish patients with hypertrophic cardiomyopathy," Annals of Medicine, vol. 46, no. 6, pp. 424-429, 2014.

[3] P. Jääskeläinen, T. Heliö, K. Aalto-Setälä et al., “Two founder mutations in the alpha-tropomyosin and the cardiac myosinbinding protein $\mathrm{C}$ genes are common causes of hypertrophic cardiomyopathy in the Finnish population," Annals of Medicine, vol. 45, no. 1, pp. 85-90, 2013.

[4] D. J. Duncker, J. Bakkers, B. J. Brundel, J. Robbins, J. C. Tardiff, and L. Carrier, "Animal and in silico models for the study of sarcomeric cardiomyopathies," Cardiovascular Research, vol. 105, no. 4, pp. 439-448, 2015.

[5] K. Takahashi, K. Tanabe, M. Ohnuki et al., "Induction of pluripotent stem cells from adult human fibroblasts by defined factors," Cell, vol. 131, no. 5, pp. 861-872, 2007.

[6] A. L. Lahti, V. J. Kujala, H. Chapman et al., "Model for long QT syndrome type 2 using human iPS cells demonstrates arrhythmogenic characteristics in cell culture," Disease Models and Mechanisms, vol. 5, no. 2, pp. 220-230, 2012.

[7] A. Moretti, M. Bellin, A. Welling et al., "Patient-specific induced pluripotent stem-cell models for long-QT syndrome," The New England Journal of Medicine, vol. 363, no. 15, pp. 1397-1409, 2010.

[8] A. L. Kiviaho, A. Ahola, K. Larsson et al., "Distinct electrophysiological and mechanical beating phenotypes of long QT syndrome type 1-specific cardiomyocytes carrying different mutations," IJC Heart \& Vasculature, vol. 8, pp. 19-31, 2015.

[9] K. Kujala, J. Paavola, A. Lahti et al., "Cell model of catecholaminergic polymorphic ventricular tachycardia reveals early and delayed afterdepolarizations," PLoS ONE, vol. 7, no. 9, Article ID e44660, 2012.

[10] A. Fatima, G. Xu, K. Shao et al., "In vitro modeling of ryanodine receptor 2 dysfunction using human induced pluripotent stem cells," Cellular Physiology and Biochemistry, vol. 28, no. 4, pp. 579-592, 2011.

[11] N. Sun, M. Yazawa, J. Liu et al., "Patient-specific induced pluripotent stem cells as a model for familial dilated cardiomyopathy," Science Translational Medicine, vol. 4, no. 130, Article ID 130ra47, 2012.

[12] F. Lan, A. S. Lee, P. Liang et al., "Abnormal calcium handling properties underlie familial hypertrophic cardiomyopathy pathology in patient-specific induced pluripotent stem cells," Cell Stem Cell, vol. 12, no. 1, pp. 101-113, 2013.

[13] L. Han, Y. Li, J. Tchao et al., "Study familial hypertrophic cardiomyopathy using patient-specific induced pluripotent stem cells," Cardiovascular Research, vol. 104, no. 2, pp. 258-269, 2014.

[14] A. Tanaka, S. Yuasa, G. Mearini et al., "Endothelin-1 induces myofibrillar disarray and contractile vector variability in hypertrophic cardiomyopathy-induced pluripotent stem cell-derived cardiomyocytes," Journal of the American Heart Association, vol. 3, no. 6, Article ID e001263, 2014.

[15] M. Ohnuki, K. Takahashi, and S. Yamanaka, "Generation and characterization of human induced pluripotent stem cells," in Current Protocols in Stem Cell Biology, chapter 4, unit 4A.2, John Wiley \& Sons, 2009.

[16] M. Ojala, K. Rajala, M. Pekkanen-Mattila, M. Miettinen, H. Huhtala, and K. Aalto-Setälä, "Culture conditions affect cardiac differentiation potential of human pluripotent stem cells," PLoS ONE, vol. 7, no. 10, Article ID e48659, 2012.

[17] R. J. Lund, T. Nikula, N. Rahkonen et al., "High-throughput karyotyping of human pluripotent stem cells," Stem Cell Research, vol. 9, no. 3, pp. 192-195, 2012.

[18] C. Mummery, D. Ward-van Oostwaard, P. Doevendans et al., "Differentiation of human embryonic stem cells to cardiomyocytes: role of coculture with visceral endoderm-like cells," Circulation, vol. 107, no. 21, pp. 2733-2740, 2003.

[19] O. P. Hamill, A. Marty, E. Neher, B. Sakmann, and F. J. Sigworth, "Improved patch-clamp techniques for high-resolution current recording from cells and cell-free membrane patches," Pflügers Archiv European Journal of Physiology, vol. 391, no. 2, pp. 85-100, 1981.

[20] J. Rae, K. Cooper, P. Gates, and M. Watsky, "Low access resistance perforated patch recordings using amphotericin B," Journal of Neuroscience Methods, vol. 37, no. 1, pp. 15-26, 1991.

[21] K. J. Livak and T. D. Schmittgen, "Analysis of relative gene expression data using real-time quantitative PCR and the $2^{-\Delta \Delta C_{\mathrm{T}}}$ method," Methods, vol. 25, no. 4, pp. 402-408, 2001.

[22] A. Ahola, A. L. Kiviaho, K. Larsson, M. Honkanen, K. AaltoSetälä, and J. Hyttinen, "Video image-based analysis of single human induced pluripotent stem cell derived cardiomyocyte beating dynamics using digital image correlation," BioMedical Engineering Online, vol. 13, article 39, 2014.

[23] J. C. Tardiff, L. Carrier, D. M. Bers et al., "Targets for therapy in sarcomeric cardiomyopathies," Cardiovascular Research, vol. 105, no. 4, pp. 457-470, 2015.

[24] C. Dambrot, S. R. Braam, L. G. J. Tertoolen, M. Birket, D. E. Atsma, and C. L. Mummery, "Serum supplemented culture medium masks hypertrophic phenotypes in human pluripotent stem cell derived cardiomyocytes," Journal of Cellular and Molecular Medicine, vol. 18, no. 8, pp. 1509-1518, 2014.

[25] S. J. van Dijk, E. R. Paalberends, A. Najafi et al., "Contractile dysfunction irrespective of the mutant protein in human hypertrophic cardiomyopathy with normal systolic function," Circulation: Heart Failure, vol. 5, no. 1, pp. 36-46, 2012.

[26] V. Sequeira, P. J. M. Wijnker, L. L. A. M. Nijenkamp et al., "Perturbed length-dependent activation in human hypertrophic cardiomyopathy with missense sarcomeric gene mutations," Circulation Research, vol. 112, no. 11, pp. 1491-1505, 2013.

[27] N. Frey, M. Luedde, and H. A. Katus, "Mechanisms of disease: hypertrophic cardiomyopathy," Nature Reviews Cardiology, vol. 9, no. 2, pp. 91-100, 2012. 
[28] B. J. Maron, "Hypertrophic cardiomyopathy: a systematic review," The Journal of the American Medical Association, vol. 287, no. 10, pp. 1308-1320, 2002.

[29] P. Liang, F. Lan, A. S. Lee et al., "Drug screening using a library of human induced pluripotent stem cell-derived cardiomyocytes reveals disease-specific patterns of cardiotoxicity," Circulation, vol. 127, no. 16, pp. 1677-1691, 2013.

[30] P. Jääskeläinen, R. Miettinen, P. Kärkkäinen, L. Toivonen, M. Laakso, and J. Kuusisto, "Genetics of hypertrophic cardiomyopathy in eastern Finland: few founder mutations with benign or intermediary phenotypes," Annals of Medicine, vol. 36, no. 1, pp. 23-32, 2004.

[31] P. Jääskeläinen, J. Kuusisto, R. Miettinen et al., "Mutations in the cardiac myosin-binding protein $\mathrm{C}$ gene are the predominant cause of familial hypertrophic cardiomyopathy in eastern Finland," Journal of Molecular Medicine, vol. 80, no. 7, pp. 412-422, 2002.

[32] S. Marston, O. Copeland, A. Jacques et al., "Evidence from human myectomy samples that MYBPC3 mutations cause hypertrophic cardiomyopathy through haploinsufficiency," Circulation Research, vol. 105, no. 3, pp. 219-222, 2009.

[33] W. Rottbauer, M. Gautel, J. Zehelein et al., "Novel splice donor site mutation in the cardiac myosin-binding protein- $\mathrm{C}$ gene in familial hypertrophic cardiomyopathy. Characterization of cardiac transcript and protein," Journal of Clinical Investigation, vol. 100, no. 2, pp. 475-482, 1997.

[34] S. J. Van Dijk, D. Dooijes, C. Dos Remedios et al., "Cardiac myosin-binding protein $\mathrm{C}$ mutations and hypertrophic ardiomyopathy haploinsufficiency, deranged phosphorylation, and cardiomyocyte dysfunction," Circulation, vol. 119, no. 11, pp. 1473-1483, 2009.

[35] A. S. Helms, F. M. Davis, D. Coleman et al., "Sarcomere mutation-specific expression patterns in human hypertrophic cardiomyopathy," Circulation: Cardiovascular Genetics, vol. 7, no. 4, pp. 434-443, 2014.

[36] J. L. Theis, J. M. Bos, J. D. Theis et al., "Expression patterns of cardiac myofilament proteins: genomic and protein analysis of surgical myectomy tissue from patients with obstructive hypertrophic cardiomyopathy," Circulation: Heart Failure, vol. 2, no. 4, pp. 325-333, 2009. 

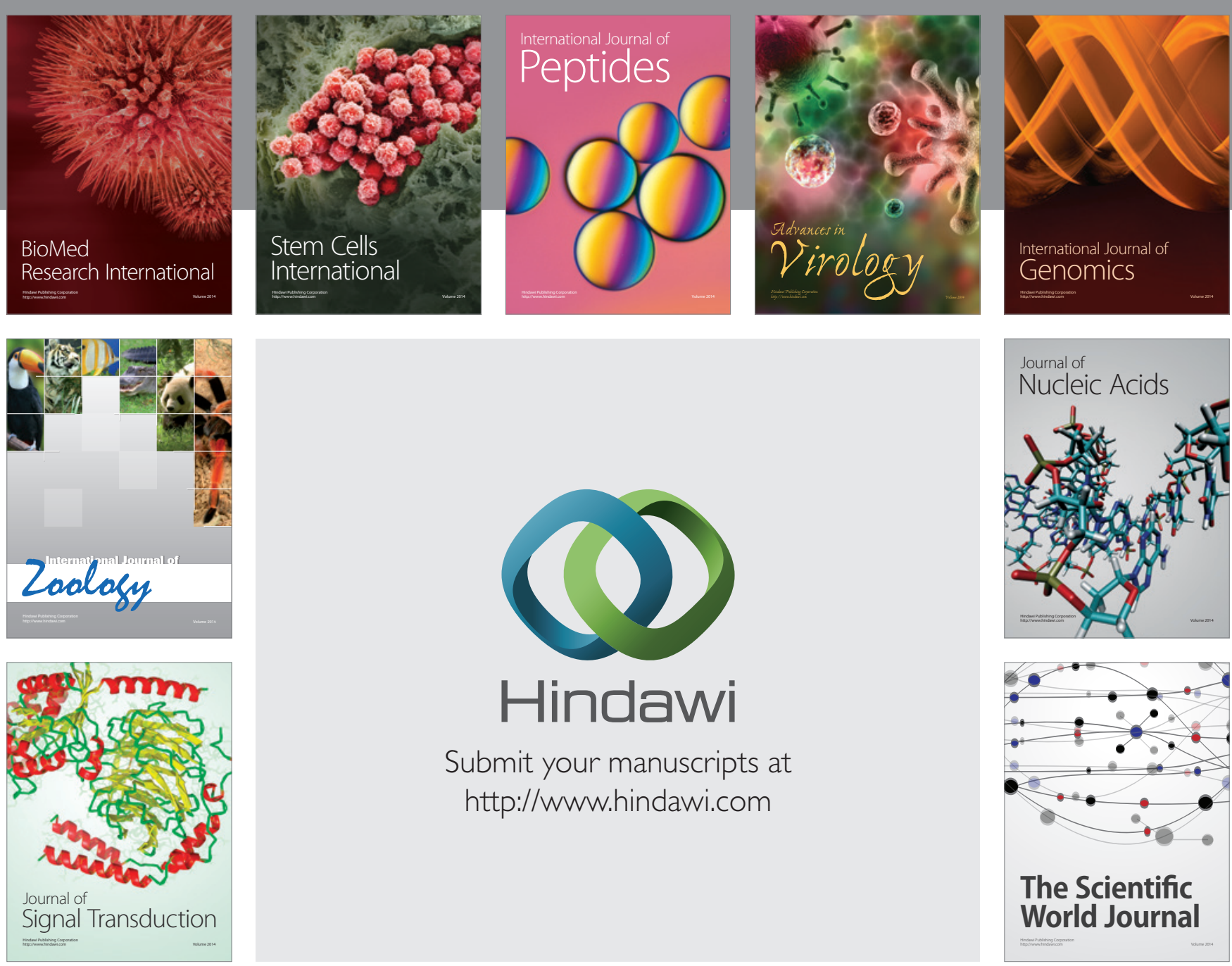

Submit your manuscripts at

http://www.hindawi.com
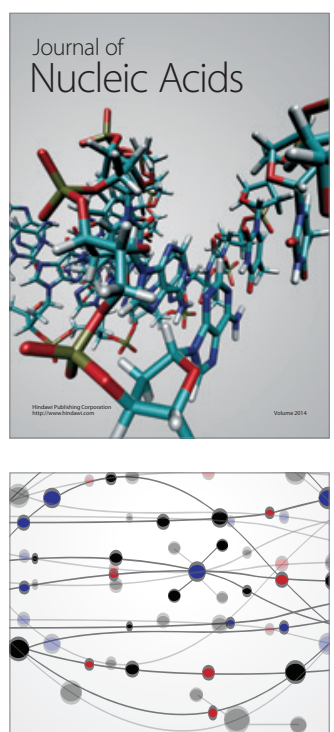

The Scientific World Journal
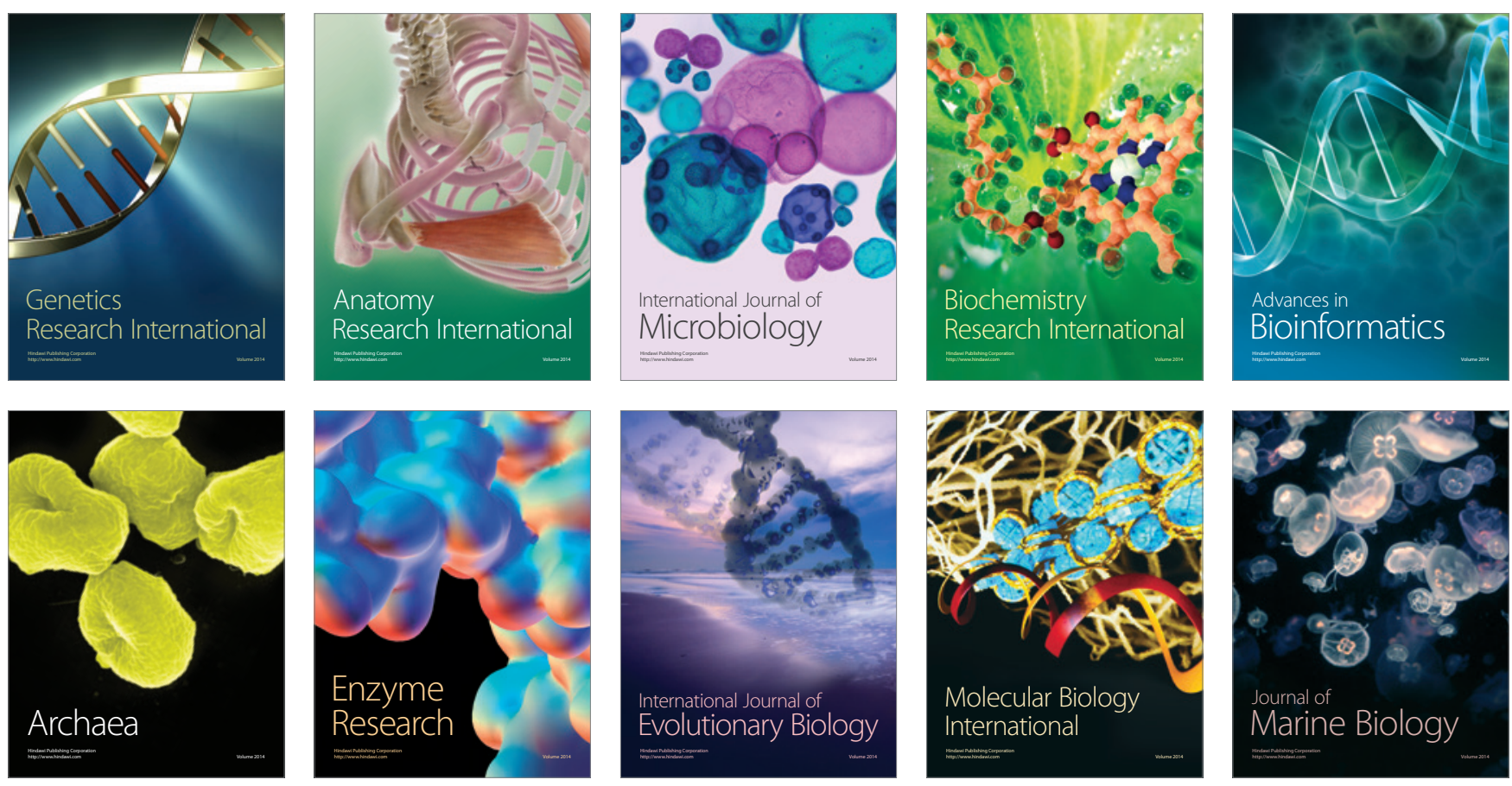